\title{
Una possibile conferma postuma: punti di congiunzione tra Schopenhauer e Darwin
}

\author{
Uma possível confirmação póstuma: pontos de contato entre \\ Schopenhauer e Darwin \\ Fiorella Giaculli \\ Pós-Graduanda em Filosofia pela Università degli Studi di Napoli "Federico II" \\ E-mail: fioregiaculli@gmail.com
}

Riassunto: Il presente saggio illustra alcuni tratti in comune tra il pensiero del filosofo e del naturalista: il rifiuto della spiegazione teologica della natura, l'idea di lotta per l'esistenza, l'importanza della conservazione, l'unità della discendenza, le somiglianze organiche, intellettive ed emotive tra i viventi. Nel contempo, considera alcuni aspetti della teoria darwiniana come possibile «riaffermazione» della interpretazione schopenhaueriana della natura.

Palavras-chave: Schopenhauer; Darwin; Vita; Natura; Dialogo tra filosofia e biologia.
Abstract: 0 ensaio sublinha alguns traços em comum entre o pensamento do filósofo e do naturalista: a rejeição da explicação teológica da natureza, a ideia de luta pela existência, a importância da conservação, a unidade da descendência, as semelhanças orgânicas, mentais e afetivas entre os seres vivos. Ao mesmo tempo, analisa alguns aspectos da teoria darwiniana como possível «reafirmação» da interpretação schopenhaueriana da natureza.

Keywords: Schopenhauer; Darwin; Vida; Natureza; Diálogo entre filosofia e biologia. 


\section{Una breve introduzione}

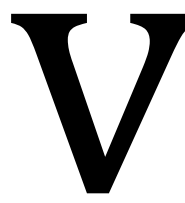

oler disegnare dei punti di contatto tra il pensiero schopenhaueriano e quello darwiniano potrebbe risultare un proposito non appropriato agli studiosi in questione per varie ragioni.

Basti pensare che Schopenhauer non ha incontrato Darwin nel corso della sua vita - la terza edizione de Il mondo come volontà e rappresentazione viene pubblicata lo stesso anno della prima de L'origine delle specie, 1859, e l'anno seguente il filosofo muore, avendo letto soltanto un «dettagliato riassunto pubblicato nel 'Times' ${ }^{1}$ » - e viceversa, non si hanno testimonianze di letture schopenhaueriane da parte di Darwin. Si tenga conto della questione eidetica del tutto ignota, (o forse ignorata perché inconcepibile) al naturalista inglese, che squadrava fenomeni soggetti al tempo, allo spazio e alla causalità, contemplando «ogni tipo di somiglianza», e che tentava di comprendere viventi remoti attraverso la frammentaria documentazione fossile. Si badi anche alla profonda crepa tra i due circa la considerazione delle specie. Occorre pronunciare due attributi antitetici per comprendere la portata del divario: per Schopenhauer le specie sono «fisse ${ }^{2} » ;$ per Darwin le specie «non sono immutabili», considerazione che fu come «confessare un omicidio ${ }^{3} »$. Ma fu anche un pensiero opposto a quello iniziale. Quindi, non escludiamo che il filosofo, proseguendo con le indagini naturalistiche, avrebbe potuto rivalutare quanto scoperto, riducendo indirettamente la distanza con lo scienziato inglese.

Questi sono alcuni aspetti che, a nostro avviso, costituiscono elementi di divergenza, e che potrebbero non giustificare o non rendere comprensibile l'intento di illustrare i punti di contatto tra i due studiosi della natura. Aspetti che abbiamo anteposto per dedicarci, quasi esclusivamente, a quei tratti che li congiungono, nonostante le differenze.

Il proponimento di esporli ed evidenziarli muove da un elemento in particolare: il sentimento di «fiducia» che Schopenhauer ripone nelle scienze naturali future, quali

\footnotetext{
${ }^{1}$ Riassunto che definisce nei termini di «platter Empirismus», in una lettera del marzo 1860 indirizzata ad Adam von Doss. Cfr. SCHOPENHAUER, A. Gesammelte Briefe, p. 472. In: VOLPICELLI, I. Schopenhauer. La natura vivente e le sue forme, p. 37.

2 SCHOPENHAUER, A. P II, p. 202.

${ }^{3}$ Cfr. DARWIN, C. Lettera a J. D. Hooker, 11 gennaio 1844. In: DARWIN, C. L'origine delle specie. Abbozzo del 1842. Lettere 1844-1858. Comunicazione del 1858, p. 71.

Una possibile conferma postuma: punti di congiunzione tra Schopenhauer e Darwin
} 
possibili saperi che adducono ulteriori riprove alla sua filosofia (in aggiunta a quelle da lui presentate, soprattutto e sistematicamente, in Sulla volontà nella natura. Dissertazione sulle conferme che la filosofia dell'autore ha ricevuto, dal suo apparire, dalle scienze empiriche. Titolo che riassume emblematicamente il contenuto dello scritto).

Ecco come esplica tale sentire nella Conclusione del libro appena menzionato:

Accanto alle conferme certamente vistose enumerate in questo trattato, che le scienze empiriche hanno fornito alla mia dottrina dalla sua apparizione, ma indipendentemente da essa, se ne allineano senza dubbio ancora molte, di cui non sono venuto a conoscenza; giacché quanto esigua è la parte della letteratura sulle scienze della natura, così attivamente coltivata in tutte le lingue, che tempo, occasione e pazienza permettono al singolo di conoscere! Ma anche già quanto qui comunicato mi dà fiducia che il tempo maturi venendo incontro alla mia filosofia, e con una gioia che mi rincuora vedo come nel corso degli anni le scienze empiriche si presentino a poco a poco come testimoni non sospetti per una dottrina sulla quale i «filosofi di professione» hanno osservato un silenzio diplomatico, inviolabile $[. . .]^{4}$.

Quindi, il confronto tra Schopenhauer e Darwin non mira semplicemente a descrivere le affinità tra i due studiosi, poste e comprese le dissimilarità; né intende presentare il filosofo di Danzica come precursore di Darwin, meno che mai come «evoluzionista» ${ }^{5}$. Vorrebbe soprattutto, nell'osservare le somiglianze tra i due, mettere in risalto le riaffermazioni che a nostro avviso la metafisica schopenhaueriana avrebbe potuto ricevere dalla scienza darwiniana.

Pertanto, abbiamo riflettuto su alcuni nuclei concettuali che avvicinano i due pensatori, e che ribadiscono biologicamente ciò che è stato compreso filosoficamente. Li enunciamo dapprima concisamente, per trattarli estesamente poi: la non condivisione della spiegazione teologica della natura, con le innumerevoli implicazioni che comporta; la contrapposizione a essa di una teoria della discendenza; l'unità e identità nella

\footnotetext{
${ }^{4}$ SCHOPENHAUER, A. N, p. 231.

${ }^{5}$ Questa tesi è sostenuta ad esempio da LOVEJOY, A. O. Schopenhauer as an evolutionist. In: GLASS OWSEI TEMKIN, H. B., STRAUSS, W. L. Forerunners of Darwin 1745-1859. Icilio Vecchiotti accenna ad un Simmel che ha riconosciuto in Schopenhauer elementi evoluzionisti (Cfr. VECCHIOTTI, I. Introduzione a Schopenhauer, p. 135) e Antonio Bellingreri fa riferimento a Vattimo e Hübscher che hanno segnalato all'incirca la stessa cosa (Cfr. BELLINGRERI, A. La metafisica tragica di Schopenhauer, pp. 49, 63.). Anche Segala osserva che Schopenhauer è stato considerato sia il precursore dell'evoluzionismo che della psicoanalisi (Cfr. SEGALA, M. Schopenhauer, la filosofia, le scienze, p. 12). Baccelli invece si sofferma sulle somiglianze che intercorrono tra Schopenhauer, Darwin e Bergson intorno all'idea di vita (Cfr. BACCELLI, V. Evoluzione e vita in Schopenhauer e Bergson). 
diversità, sebbene in accezioni dissimili; la rilevanza della lotta per l'esistenza; la centralità dell'adattamento e della conservazione; la considerazione degli animali non umani quali esseri dotati di intelligenza e emozioni; la persistenza nel ricercare unita all’umiltà di riconoscere i limiti dello scandagliare.

\section{Il comune distanziarsi dall'interpretazione teologica della natura}

Nell'introduzione a L'origine delle specie, Darwin dichiara di essere giunto alla conclusione che le specie «non sono state create indipendentemente, ma sono discese, come se si trattasse di varietà, da altre specie ${ }^{6} . »$ È una considerazione che non sottende, almeno fino alla sesta edizione de L'origine, un'esclusione totale dell'elemento divino, il quale appare fino ad allora come una sorta di soffio primordiale, che infonde la vita per la prima volta, la quale sarebbe proseguita da sé, secondo le leggi individuate da Darwin7. Anche quell'anelito residuale sarebbe scomparso.

Secondo il naturalista, la spiegazione teologica non è, de facto, una spiegazione, poiché qualsiasi esistente è ricondotto alla volontà del creatore con la quale si dovrebbe esplicare ogni questione e dipanare ogni dubbio. Si ha soltanto la riaffermazione di una condizione, senza rischiarala in alcun modo: «chi crede in atti di creazione separati e innumerevoli dirà che [in questi casi] così è piaciuto al creatore che un essere di un certo tipo prendesse il posto di uno di un altro tipo; a me, però, sembra che così ci si limiti a riaffermare lo stesso fatto in un linguaggio solenne ${ }^{8} . »$ Per giunta, servirsi dell'“dimostrazione"-asserzione teologica è una maniera per mascherare l'ignoranza con la presunta onnipotenza del sommo creatore, anziché dedicarsi a quanto sia stato scorto e nel contempo riconoscere i limiti della conoscenza umana.

A riguardo, Darwin riscontra che

è tanto facile nascondere la nostra ignoranza dietro espressioni come 'piano della creazione', 'unità di disegno' ecc. e pensare che in questo modo abbiamo spiegato un fatto, mentre in realtà lo abbiamo soltanto riaffermato. Chi è portato ad attribuire più peso alle difficoltà inspiegate che alla spiegazione di un certo numero di fatti, respingerà sicuramente

\footnotetext{
${ }^{6}$ DARWIN, C. L'origine delle specie, p. 9. D'ora in avanti lo scritto sarà indicato come L'origine.

${ }^{7}$ Cfr. Ivi, p. 515. Cfr. inoltre DARWIN, C. Lettere sulla religione, pp. 23-28.

8 DARWIN, C. L'origine, p. 202.

Una possibile conferma postuma: punti di congiunzione tra Schopenhauer e Darwin
} 
la mia teoria 9 .

Che per Darwin ci sia un'unità nella natura è indiscutibile, ma la natura di quell'unità è ben lungi dall'avere sembianze teomorfe. Il carattere unitario è dato dalla discendenza comune con modificazione.

Inoltre, quand'anche si volesse ammettere l'ipotesi di un'unità armonica derivante da un essere sommamente buono e giusto, che ha provveduto a garantire la più perfetta concordanza tra le strutture e gli abiti, gli organi e le funzioni, le relazioni con altri viventi, comunque non si capirebbero le cause di innumerevoli situazioni: ad esempio, perché molte parti animali sono inutili? Perché il vitello allo stato embrionale ha già i denti e alcuni tipi di coleotteri hanno le ali avvizzite conservate sotto una copertura? L'intelligent designer ha progettato così, ripeterebbe la stesso ritornello. Di contro, l'«evoluzionista riluttante» osserva che «la natura si è preoccupata di rivelare, attraverso gli organi rudimentali e le strutture omologhe, lo schema da essa seguito nelle modificazioni; ma, a quanto pare, noi ci rifiutiamo ostinatamente di capirlo ${ }^{10} . »$

Come si spiegherebbero i ritorni ad alcuni caratteri da tempo perduti? L'occasionale comparsa di strisce sulle zampe e sulle spalle di diverse specie del genere del cavallo non è motivata teologicamente. Fatto che può invece essere chiarito con la teoria per cui queste specie sono discese da un progenitore a strisce, di cui hanno ereditato alcuni caratteri ${ }^{11}$.

Ancora, l'ipotesi creazionista, come giustificherebbe le cospicue tonalità cromatiche delle conchiglie, degli uccelli, delle piante? Assicurerebbe che le conchiglie dai colori brillanti sono state create per un mare caldo, quelle dai colori più opachi per un mare freddo etc. Viceversa, per Darwin, quelle colorazioni sono variazioni legate all'azione cumulativa di selezione naturale e condizioni di vita.

Soprattutto, perché mai un Dio onnipotente e buono non avrebbe potuto orientare benevolmente leggi naturali e comportamenti animali? In quanto Dio, non avrebbe dovuto prevenire alcune inclinazioni e atrocità, provando a ridurre l'elemento doloroso della vita?

L'inadeguata interpretazione teologica della genesi naturale, unitamente al dolore mondano che un Dio, se esistesse, dovrebbe lenire, costituiscono per Darwin motivi

\footnotetext{
${ }^{9}$ Ivi, p. 506.

10 Ivi, p. 505.

11 Cfr. Ivi, p. 497.

Una possibile conferma postuma: punti di congiunzione tra Schopenhauer e Darwin
} 
sufficienti per respingere argomentazioni fisico-teologiche.

Ora, sia ben chiaro, il prendere le distanze da cotali posizioni non implica l'esclusione perentoria di una causa prima, trascendente, inconoscibile per l'intelletto umano ma non per questo giudicabile inesistente indubitabilmente.

Darwin avrebbe allontanato, sempre con maggiore convinzione, tracce divine dalla natura: muovendo dall'idea di un creatore della prima forma di vita, o delle poche prime; dichiarando per lo più l'impossibilità di una conoscenza sovrasensibile, definendosi per questo agnostico; giungendo a escludere qualsiasi elemento trascendente.

Emblematica del primo pensiero è, come abbiamo accennato, la chiusa de L'origine, in cui l'elemento divino si configura come $\pi v \varepsilon \tilde{u} \mu \alpha$. Paradigmatica per i vari elementi che lo schiererebbero contra l'esistenza di un creatore è una lettera del '60 al caro Gray, significativa anche perché mostra il bisogno di conferme tipico di Darwin, senza le quali non era solito pronunciarsi con convinzione. Ragion per cui, mancando ogni certezza a riguardo, il definirsi «agnostico» costituisce il termine più appropriato per descrivere il suo «stato mentale ${ }^{12}$ », caratterizzato anche dall'idea che occorre separare la sfera scientifica da quella religiosa.

Per una maggiore intelligibilità della questione, riportiamo la summenzionata lettera quasi nella sua interezza, illuminando la tematica senza necessitare di commenti esplicativi:

[...] Per quanto riguarda la prospettiva teologica della questione, si tratta di un tema sempre penoso per me. - Sono confuso. - Non avevo alcuna intenzione di scrivere da ateo. Ma riconosco che non riesco a vedere, con la stessa semplicità di altri, e come vorrei riuscire a fare, le prove del disegno e della benevolenza [divini] tutt'attorno a noi. Mi sembra che nel mondo vi sia troppa miseria. Non riesco a persuadermi del fatto che un Dio benevolo e onnipotente abbia creato di proposito gli Ichneumonidae con la precisa intenzione che si nutrissero del corpo dei bruchi ancora vivi, divorandolo dall'interno; o che un gatto dovesse giocare con i topi. Non credendo questo, non vedo alcuna necessità di credere che l'occhio sia stato espressamente progettato. D'altro canto, io non posso comunque contentarmi di considerare questo meraviglioso universo, e soprattutto la natura dell'uomo, e di concludere che ogni cosa è il risultato della forza bruta. Sono incline a pensare che tutto sia il risultato di leggi progettate, mentre i dettagli, nel bene e nel male, sarebbero lasciati al lavorio di quello che possiamo chiamare caso. Non

${ }^{12}$ Cfr. La lettera indirizzata a J. Fordyce, il 7 maggio 1879. In: DARWIN, C. Lettere sulla religione, p. 112.

Una possibile conferma postuma: punti di congiunzione tra Schopenhauer e Darwin 
che questa idea mi soddisfi completamente. Sento, nel profondo, che questa materia è nel suo insieme troppo astrusa per l'intelletto umano. [...] Sono certamente d'accordo con voi, le mie idee non sono affatto necessariamente atee. Il fulmine uccide un uomo, sia esso buono o malvagio, a causa dell'azione straordinariamente complessa delle leggi naturali- un bambino (che potrebbe poi rivelarsi un idiota) nasce per azione di leggi ancora più complesse - ed io non riesco a vedere alcuna ragione perché un uomo o un altro animale non possa essere stato prodotto, originariamente, da altre leggi; e che tutte queste leggi possano essere state espressamente progettate da un Creatore onnisciente, che prevede ogni evento e conseguenza futuri. Ma più ci penso, e più cado nello sconcerto; in effetti l'ho dimostrato in questa lettera $[\ldots]^{13}$.

Simbolo del pensiero-approdo ultimo potrebbe dirsi una lettera del 1880 , in cui afferma che gli duole informare di non credere «nella Bibbia come rivelazione divina e pertanto nemmeno in Gesù Cristo come figlio di Dio ${ }^{14}$ ».

Dunque, dapprima ammettendo un soffio divino, poi sospendendo il giudizio, disgiungendo il piano religioso da quello naturalistico e pregando di rispettarlo, alfine contrastando l'ipotesi creazionista, Darwin lentamente giunge a escludere qualsiasi forma teomorfa, invitando a stendere un velo di «riverente silenzio» sull'origine primigenia.

Anche per il filosofo di Danzica la presunta spiegazione fisico-teologica è un'asserzione non argomentativa, incongruente con il circostante, oltre a essere un'ipotesi escludibile, impensabile per la sola presenza del dolore nel mondo.

Non esiste alcun Dio che genera, esterno alla natura, che si occupa del proprio creato; esiste la selezione naturale che conserva ogni variazione vantaggiosa per ciascuna specie, avendo cura di ogni suo essere.

Non esiste un Dio onnipotente che crea finalisticamente; onnipotente è solo la volontà, creatrice e creantesi, che plasma in funzione del suo conato fondamentale, l'impulso a conservarsi. Selbsterhaltung. Interna, intima essenza delle cose.

Eppure, secondo Schopenhauer, «I nostri filosofi da strapazzo prendono Dio come se fosse noto, e da lui argomentano il mondo. Con ciò essi credono di aver fatto qualche cosa. Mentre il suddetto, sia per la sua existentia che per la sua essentia è del tutto $=\mathrm{x}$, quindi una semplice parola ${ }^{15}$ ». Precisamente, «chiamare il mondo Dio non significa

\footnotetext{
13 Ivi, pp. 46-47.

${ }^{14}$ Ivi, p. 116

15 SCHOPENHAUER, A. O si pensa o si crede. Scritti sulla religione, posizione 781. - Chiaramente si tratta di una raccolta di pensieri sulla religione, provenienti da vari scritti, e non di un'opera così architettata.

Una possibile conferma postuma: punti di congiunzione tra Schopenhauer e Darwin
} 
spiegarlo: esso rimane un enigma sotto questo nome come sotto l'altro ${ }^{16}{ }^{\prime}$.

Del resto, un enigma permarranno sempre l'esistenza, l'essenza e l'origine del mondo, Daseyn, Wesen und Ursprung der Welt, poiché una conoscenza assoluta è impossibile. Pertanto, un'indagine filosofica «onesta», memore di ciò, prova a «scavare» fin dove è consentito, e non a «coprire» con parole vuote quanto è inafferrabile. L'intima essenza delle cose, a prescindere dal suo apparire «nel mondo, come mondo», non è indagabile, essendo la conoscenza per sua natura fenomenica, necessitante forme mondane, senza le quali non può ergersi né aggirarsi. A ciò si aggiunga che l'in sé della natura è privo di conoscenza, erkenntnißlos. Essa sopraggiunge come accidente, strumento, ausilio, per gettare sprazzi di luce nell'oscurità. L'autocoscienza di sé, del proprio corpo, rivela che il suo intimo fondamento è estraneo a elementi noematici, ed è

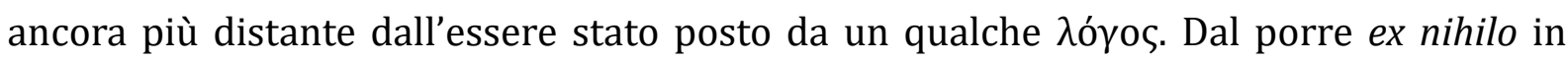
generale. Essendo ciò che per primo pone.

Non c'è alcun intervento esterno, metasensibile. La volontà è la spinta originaria verso l'esistenza, lo Streben inesausto che caratterizza ogni forma naturale, inorganica e organica; mutando incredibilmente la figura in cui si manifesta, ma non l'intimo quid di ciascuna; cambiando gli accidenti, le qualità secondarie, ma non la sostanza, comune a ogni fenomeno; variando il tipo di causa che occasiona l'agire, le innumerevoli gradazioni della coscienza, ma non il fondamento di tutte le cose, che ovunque brama la stessa cosa: la vita.

Ricapitolando con Schopenhauer:

il mondo non è stato fatto con l'ausilio della conoscenza, e quindi anche non dal difuori ma dal didentro; [...] Per quanto il pensiero fisicoteologico che debba essere stato un intelletto a ordinare e a modellare piaccia facilmente alla mente rozza, esso rimane comunque fondamentalmente storto. Giacché l'intelletto ci è noto solo dalla natura animale, quindi come un principio del tutto secondario e subordinato nel mondo, un prodotto di origine tardissima; giammai esso può essere stato la condizione della sua esistenza, né può un mundus intellegibilis precedere il mundus sensibilis, dato che solo da questo quello riceve il suo materiale. Non un intelletto ha prodotto la natura, bensì la natura l'intelletto. Ben si presenta invece la volontà, che tutto riempie e si palesa immediatamente in ogni cosa, in tal modo indicandolo come suo fenomeno, dappertutto come la cosa originaria. E perciò appunto tutti i

Pertanto, le fonti effettive del materiale raggruppato sono in parte opere che egli stesso pubblicò, in parte materiale postumo, come il Nachlass e i Gespräche. -

16 SCHOPENHAUER, A. WWV II, p. 2203.

Una possibile conferma postuma: punti di congiunzione tra Schopenhauer e Darwin 
fatti teleologici si possono spiegare in base alla volontà dell'essere stesso, in cui vengono trovati ${ }^{17}$.

Per Schopenhauer non esiste una teleologia intenzionale, divina, architettata in vista dei più svariati fini. Né comunque, escludendo l'essere sommo, i viventi sono da pensarsi finalisticamente, come corpi le cui parti sono in vista di uno scopo. Al contrario, è il modo di vivere che conduce alla formazione di una parte in una maniera e non in un'altra. Nei termini già aristotelici, lamarckiani e darwiniani poi, con le dovute distinzioni e precisazioni, è la funzione che crea l'organo e non viceversa, l'organo che crea la funzione. È la volontà, nell'accezione di necessità di sopravvivere e conservarsi rispetto a precise circostanze e abiti, che conduce a strutture, forme e funzionamenti adeguati a quell'aspirazione. Essa costituisce il da cui primigenio, il di cui, sostrato di tutti i fenomeni; è l'«animale originario», nel significato di condizione metafisica per la nascita di tutti gli altri. È il donde archetipico ed escatologico. Il che cosa che spiega l'esistenza. È la natura in cui la volontà si oggettiva e si rivela come «maestro», «materia» e «opera d'arte». Per questo il Wille è onnipotente, e non il Dio. La volontà omnia potest, non il Deus. Nella decifrazione schopenhaueriana non c'è posto per il Paradiso, o qualcosa di affine, non solo perché la conformazione mondana è ben lungi dal potersi considerare un'epifania, per ragioni fisiche ed etiche, ma soprattutto perché risulterebbe eccedente, trovando ogni cosa un'esauriente spiegazione nel volere.

Ebbene, possiamo dire quello che egli ha detto di Kant, cioè che «ha eliminato il teismo dalla filosofia, giacché in essa, come scienza e non dottrina di fede, può trovar posto solo quello che o è empiricamente dato o è stabilito con prove durevoli18.» Il motivo di tale eliminazione risiede anche, come esponevamo per Darwin, nel sentire il dolore del mondo, nell'osservare atrocità che, sebbene per una parte insite nell'irruente natura della vita (o almeno giudicata tale dallo sguardo umano) per un'altra sarebbero dovute essere attenuate dal Deus perfectissimus.

Emblematico di questa concezione è un passo dei manoscritti postumi: «Se un dio ha fatto questo mondo, io non vorrei essere quel dio, perché il dolore del mondo mi strazierebbe il cuore ${ }^{19} . »$ Per Schopenhauer la sofferenza mondana, la lotta tra individui

\footnotetext{
17 SCHOPENHAUER, A. N, pp. 100- 101.

18 SCHOPENHAUER, A. WWV I, p. 973. Per altre osservazione schopenhaueriane intorno alla confutazione kantiana della prova ontologica dell'esistenza di Dio, cfr. Ivi, Appendice. Critica della filosofia kantiana, pp. 973-981.

${ }^{19}$ SCHOPENHAUER, A. O si pensa o si crede. Scritti sulla religione, posizione 514.

Una possibile conferma postuma: punti di congiunzione tra Schopenhauer e Darwin
} 
in termini organici, sociali ed etici, l'essenza stessa della vita, costituiscono riprove della contraddizione tra un Dio magnanimo e il suo creato. Se un Dio fosse realmente tale, se esistesse, proverebbe a bilanciare il più possibile le tonalità del dolore, non facendo riporre fiducia in un luogo altro, bensì agendo nell'unico spazio che l'umano abita. Ma Dio non esiste. Il mondo è oggettivazione del caso e della volontà. Così rammenta l'incipit dei Supplementi: «Nello spazio infinito innumerevoli sfere luminose, intorno a ciascuna delle quali ne gira una dozzina circa di più piccole, illuminate, che, internamente calde, sono ricoperte da una crosta rappresa, fredda, su cui un rivestimento di muffa ha generato esseri viventi e conoscenti: è questa la verità empirica, il reale, il mondo ${ }^{20} . »$ È un mondo senza Dio.

\section{Verso l'ipotesi della discendenza}

Esclusa la validità di una spiegazione teologica della natura, i due pensatori contrappongono a essa l'ipotesi della discendenza. L'origine delle vite non è improvvisa, emanata dall'alto, da un totalmente altro. Non è esterna, esterni sono soltanto le relazioni tra i viventi, l'ambiente e i modi di vivere (certo, con inevitabili ricadute sulle forme e funzioni interne, ma questo è un altro discorso); interno è il principio creatore e conservatore delle cose che quasi coincide con il creato e il conservato. Sul «regno delle madri dell'essere» non è permesso esprimersi, ma su quanto da esso presumibilmente proviene è possibile sussurrare qualcosa, mirando e rimirando le res cosmiche, osservandole nel loro permanere e variare, sorgere e tramontare, tentando di intravedere nei loro corpi tracce primigenie, orme perenni.

Cerchiamo di capire in che modo i due pensatori contemplano la questione.

Sintetizzare i lenti e graduali passaggi mediante i quali Darwin perviene alla sua teoria, come pure dispiegarla nella sua pluralità di aspetti, richiede uno spazio che non possiamo ritagliare (lo stesso dicasi per l'idea schopenhaueriana di natura, di cui sono esposti i tratti ineludibili e quelli posti in relazione con Darwin).

Quindi, proviamo a indugiare sui punti cruciali dell'argomentazione, che emergono fin dal titolo dell'opera: On the origin of species by means of natural selection, or the preservation of favoured races in the struggle for life. Ci riferiamo ovviamente alla

20 SCHOPENHAUER, A. WWV II, p. 1023.

Una possibile conferma postuma: punti di congiunzione tra Schopenhauer e Darwin 
selezione naturale e alla lotta per l'esistenza. - Detto en passant, anche nel titolo dell'opera schopenhaueriana Die Welt als Wille und Vorstellung sono già espressi i termini nodali del suo pensiero. -

La conclusione che Darwin annuncia nell'introduzione, vale a dire che le specie non sono state create indipendentemente, ma sono discese da altre specie, si radica profondamente in quei punti appena menzionati che ora proviamo a chiarire.

Innanzitutto, possiamo osservare con Pievani che Darwin presenta prima il nocciolo della sua teoria, poi si attarda sulla descrizione dei fatti, costituendo il tutto «una sola lunga argomentazione» - parimenti, unico è il pensiero schopenhaueriano, paragonato da lui stesso a un organismo -:

La mossa fu ben ponderata perché in questo modo egli sottolineò fin dall'inizio che la selezione naturale doveva essere un processo necessario date certe circostanze (le variazioni ereditabili e la lotta per l'esistenza) e che la trasmutazione delle specie nella discendenza comune poteva essere intesa solo alla luce di quel meccanismo. Subito la novità teorica, insomma, e poi la panoplia di evidenze che la corroborano: non una massa di fatti prima e poi un'ipotesi esplicativa fra tante altre. [...] Così facendo sembra voler scongiurare un'evenienza puntuale: che il lettore possa sì accettare l'evoluzione come un insieme di dati di fatto, ma non la sua spiegazione causale centrale, cioè la selezione naturale ${ }^{21}$.

Dunque, l'oggetto dei primi due capitoli, la variazione, allo stato domestico e allo stato di natura, costituisce ciò nei confronti di cui la selezione, umana e naturale, agisce.

Osservando gli animali allevati e le piante coltivate, non si può non notare come si distinguano dagli stessi individui allo stato di natura. Si pensi a fattori quali le diverse condizioni di vita, la riproduzione in cattività, le conseguenze ereditarie, il diverso accesso al cibo, alle abitudini dissimili. Soprattutto, si consideri che l'uomo, rispetto alle variazioni allo stato domestico, non produce realmente la variabilità, ma contribuisce a provocarla, esponendo gli individui a specifiche condizioni di vita, selezionando ciò che gli è più gli aggrada o gli è più utile, a partire da ciò che la natura ha prodotto ${ }^{22}$.

Credo, argomenta Darwin,

che le condizioni di vita, per la loro azione sul sistema riproduttivo, abbiano per quanto ne sappiamo la massima importanza come causa

21 PIEVANI, T. Introduzione a Darwin, pp. 74-75.

22 Per una trattazione ben più estesa e dettagliata della questione, cfr. i capitoli I e II de L'origine.

Una possibile conferma postuma: punti di congiunzione tra Schopenhauer e Darwin 
della variabilità. A differenza di quanto pensano alcuni, non credo che la variabilità sia una contingenza necessaria e inerente a qualunque circostanza e per tutti gli esseri organici. Gli effetti della variabilità sono modificati da diversi gradi di ereditarietà e reversione. La variabilità è governata da molte leggi sconosciute, in particolare da quella della correlazione della crescita. Qualcosa può essere attribuito all'azione diretta delle condizioni di vita, qualcosa dev'essere attribuito all'uso e al non uso. Il risultato finale diviene quindi infinitamente complesso. In alcuni casi non dubito che l'incrocio tra specie un tempo distinte abbia giocato un ruolo importante all'origine delle nostre produzioni domestiche ${ }^{23}$.

La riflessione darwiniana dapprima sulla variazione allo stato domestico è anche funzionale a una distinzione fondamentale, inerente a colui che seleziona.

L'uomo, nell'ambito agricolo come in quello dell'allevamento, può selezionare, unire o disgiungere ciò che ha prodotto la natura, piegando le sue res ai propri vantaggi, capricci e bisogni; tuttavia il suo fare è limitato, soprattutto dalla temporalità e dagli strumenti di cui fruisce. «Com’è breve il tempo a sua disposizione!», esclama difatti Darwin, «E dunque quanto poveri saranno i suoi risultati a confronto con quelli accumulati dalla natura nel corso di intere ere geologiche! ${ }^{24} »$.

Di contro, la natura, la selezione naturale opera in un tempo inimmaginabile per l'uomo, instancabilmente, continuamente, «giorno per giorno e ora per ora», eliminando ciò che è dannoso e conservando ciò che è utile per l'«essere di cui si cura», sia esso una parte corporea o un abito. Inoltre, non accade che favorisce una specie senza fornire qualche beneficio a quelle con cui si rapporta, siano esse amiche o nemiche. Ad esempio, negli animali sociali, la struttura individuale si adatta a vantaggio della comunità. Parimenti, la conservazione di un utile strumento di un predatore porterà al rafforzamento di uno della preda.

Così compendia Darwin: «Chiamo selezione naturale questa conservazione delle variazioni favorevoli e l'eliminazione delle variazioni nocive. Le variazioni che non sono né utili né dannose non saranno toccate dalla selezione naturale e resteranno fluttuanti, come vediamo nelle specie polimorfiche ${ }^{25}$ ». Dunque, essa conserva e somma tutto ciò che è buono, sia esso un colore, una forma, un odore, uno strumento, un organo, un abito. Scarta tutto ciò che è cattivo, svantaggioso, di ostacolo. Lavora «silenziosamente,

${ }^{23}$ Ivi, p. 51 (I corsivi sono nostri). - Per comprendere accuratamente lo svolgimento delle leggi della variazione, si legga il capitolo $\mathrm{V}$ de L'origine, a esse dedicato.

${ }^{24}$ Ivi, p. 94.

${ }^{25}$ Ivi, p. 91.

Una possibile conferma postuma: punti di congiunzione tra Schopenhauer e Darwin 
impercettibilmente, quando e dovunque se ne offra l'occasione, al miglioramento di ogni essere vivente in relazione alle sue condizioni di vita organiche e inorganiche ${ }^{26 . »}$

\section{Lotta per la vita e conservazione della vita}

La selezione naturale conserva tutto ciò che è utile per gli individui all'interno della «grande battaglia della vita», ineludibile a causa del

tasso elevato con cui tutti gli organismi tendono a crescere di numero. Ogni essere, che nel corso naturale della vita produce diverse uova o semi, dev'essere soggetto a distruzione in qualche periodo della vita, in qualche stagione o anno, altrimenti, in base al principio dell'aumento in progressione geometrica, il numero di quegli esseri diventerebbe presto così smisuratamente grande che nessun paese potrebbe sopportarlo. Dunque, poiché vengono prodotti più individui di quanti ne possono sopravvivere, dev'esserci in ogni caso una lotta per l'esistenza tra un individuo e l'altro della stessa specie, oppure tra individui di specie diverse, o contro le condizioni fisiche di vita. È la dottrina di Malthus applicata con forza molteplice all'insieme di regni animale e vegetale $[\ldots]^{27}$.

L'inesorabile lotta per l'esistenza è un'espressione dal «senso ampio e metaforico», che comprende sì una contesa, come si evince dal passaggio appena riportato, ma anche la vita dell'individuo, la sua capacità di lasciare una discendenza, e la dipendenza di ogni essere da un altro.

Stricto sensu, una lotta tra individui della stessa specie è «durissima» poiché essi abitano gli stessi luoghi, si nutrono dello stesso cibo e sono sottoposti agli stessi pericoli e bisogni. Diversamente, la lotta tra individui lontani nella scala naturae è meno severa, e possiamo immaginare perché ciò accada. Esempi della struggle for life sono due canidi che in tempo di carestia si contendono il cibo; una pianta nel deserto che lotta contro la siccità; il vischio disseminato dagli uccelli, che lotta con altre piante affinché siano i suoi propri semi ad essere più diffusi.

Lato sensu, l'espressione indica anche il collegamento «spesso nascosto» tra una preda e un predatore (si pensi alla struttura dei denti e degli artigli perfettamente conformi alle proprie prede, o alle zampe dei parassiti adeguate al pelo su cui attaccarsi) e il co-adattamento tra coloro che vivono nello stesso territorio, e che quindi hanno 
necessità affini.

Ora, poiché tutte le piante e gli animali tendono a crescere in progressione geometrica (e da questo segue un'altra accezione della struggle for life), è necessario, secondo Darwin, che tale tendenza sia controllata da qualche forma di «distruzione»; ad esempio legata al clima, al cibo, alla quantità di prole, alle epidemie, al numero di nemici nello stesso habitat. A un tempo, è necessario ci sia anche qualcosa che conservi le variazioni utili all'interno della contesa, che consenta di sopravvivere, di resistere, di reagire e contrapporsi a ciò che minaccia la propria vita. Ecco la selezione naturale 28 . Dalla contesa l'armonia.

Anche per Schopenhauer la vita è lotta, Kampf ${ }^{29}$. Certamente non è contemplato l'elemento della crescita in progressione geometrica ${ }^{30}$, ma crediamo di non esagerare nello scorgere nell'impulso vitale qualcosa di vagamente affine a essa, nella misura in cui ogni vivente «preme e spinge verso l'esistenza ${ }^{31}$ », incessantemente, per continuare a vivere, e tale brama di essere e conservarsi comporta una «sete» di materia, di spazio e di tempo, non bastevole per tutte le aspirazioni, rivolte all'immediato e all'avvenire - in quest'ultimo caso mediante il riprodursi, il continuare attraverso l'altro (in tal senso

\footnotetext{
28 «In virtù di questa lotta qualsiasi variazione, per quanto piccola e da qualunque causa proceda, se è vantaggiosa per l'individuo di una qualsiasi specie nelle sue relazioni infinitamente complesse con gli altri organismi e con la natura esterna, tenderà alla conservazione di quell'individuo e sarà generalmente ereditata dai suoi discendenti.» Ivi, p. 71.

${ }^{29}$ È interessante notare che entrambi, Schopenhauer e Darwin, mettono quasi in guardia dalla parvenza idilliaca della natura, che nasconde conflitti silenti non sempre udibili dall'umano. Il primo, in un colloquio con Frédéric Morin, tra le varie argomentazioni per cui il nostro non sarebbe il migliore dei mondi possibili, osserva che «nella foresta tranquilla, che sembra sognare in disparte, nella prateria che allieta lo sguardo del poeta, tutto è guerra intestina, sterminio implacabile, da albero a albero, da filo d'erba a filo d'erba, da fiore a fiore. Ogni radice si estende silenziosamente nell'ombra per rubare alla sua vicina l'atomo che la nutra. Il muschio e l'edera si avvinghiano intorno alla quercia per succhiarle la linfa. Osservate questa povera pianta secca e smagrita: è stata soffocata, è stata uccisa da quelle che l'attorniavano con la loro folle gelosia.» SCHOPENHAUER, A. Colloqui, p. 201. Il secondo espone qualcosa di non dissimile: «Il volto della natura ci appare splendente di gioia e spesso percepiamo una sovrabbondanza di cibo, ma non vediamo o dimentichiamo che gli uccelli che cantano oziosamente intorno a noi vivono di insetti e di semi e dunque distruggono in continuazione la vita, oppure dimentichiamo in qual misura quei canterini, le loro uova o i loro nidi sono distrutti da uccelli e animali rapaci, o non ricordiamo che, se in un certo momento il cibo è sovrabbondante, non è così in tutte le stagioni di ogni anno. [...] Il volto della natura può essere paragonato a una superficie cedevole con diecimila cunei affilati stipati l'uno vicino all'altro e spinti dentro da colpi incessanti, con alcuni cunei che, a volte, vengono colpiti con maggiore forza.» DARWIN, C. L'origine, p. 77.

${ }^{30}$ Circa questa tendenza, Darwin segnala che non si hanno eccezioni alla regola per cui ogni essere organico cresce a un tasso così elevato che, se non venisse distrutto, la terra in poco tempo sarebbe ricoperta dalla discendenza di una singola coppia. Per un'illustrazione più completa della questione, cfr. Ivi, pp. 72-77.

${ }^{31}$ SCHOPENHAUER, A. WWV II, p. 1659.

Una possibile conferma postuma: punti di congiunzione tra Schopenhauer e Darwin
} 
crescita $)^{32}$ -

Per Darwin la lotta per la vita, in un senso più stretto, deriva dal comune impulso ad aumentare di numero, in un mondo che non può accoglierlo, e che per questo la natura provvede ad arginare. Dipende anche dal fatto che tra gli individui della stessa specie i bisogni sono i medesimi, ma non sempre omogeneamente soddisfabili, quindi lo scontro per perseguire gli stessi fini è inevitabile, e la vittoria è vicendevole all'interno di un conflitto che non è senza posa.

Anche per Schopenhauer, accantonato l'elemento malthusiano che nebulosamente possiamo riscontrare nell'essenza del volere, il conflitto tra i fenomeni si origina non solo dal dissidio della volontà con se stessa, ma dal fatto che ciascuno tende a e si contende un'unica materia, non bastevole a soddisfare, ad abbracciare le uguali tensioni delle diverse forme di vita. Cambia l'aspetto in cui questo anelito e questo conflitto si esplicano, ma l'intimo quid resta invariato:

nella natura vediamo dappertutto contesa, lotta e alternarsi della vittoria [...] Ogni grado di oggettivazione della volontà contende all'altro la materia, lo spazio, il tempo. La materia deve, nella sua permanenza, mutar forma costantemente in quanto, sul filo conduttore della causalità, fenomeni meccanici, fisici, chimici e organici, affollandosi avidamente per passare avanti, si strappano reciprocamente la materia, ciascuno volendo manifestare la sua idea ${ }^{33}$.

La «lotta universale» riguarda ogni grado ${ }^{34}$ : il «mondo conglobato in una sfera» è oggettivazione della tensione tra la forza di attrazione e la forza di repulsione. La prima è forza di gravità che preme costantemente, la seconda è impenetrabilità, che, sia per rigidezza che per elasticità, resiste alla gravità. Questa correlazione di pressione e

\footnotetext{
32 Rispetto all'idea condivisa di «lotta per l'esistenza», si legga BACCELLI, V. Evoluzione e vita in Schopenhauer e Bergson, pp. 100-106. Ibidem, l'autrice accenna anche ad altre due somiglianze tra il pensiero del filosofo e del naturalista: quella tra la volontà di vivere e la selezione naturale, poiché entrambe forze conservatrici; e quella tra la riproduzione, quale principio fondamentale per la conservazione della specie, e l'istinto sessuale, quale forma di conservazione del volere. In effetti, il Wille schopenhaueriano potrebbe sì somigliare alla natural selection darwiniana, per il carattere conservativo che accomuna entrambi, tuttavia, in quanto tensione alla conservazione e al «potenziamento», potrebbe rievocare soprattutto la tendenza alla crescita di ogni vivente. In quanto anelito perenne al perdurare, è paragonabile sia alla selezione naturale sia alla tendenza a crescere di numero. 0 ancora, rimanendo nell'ambito di possibili rassomiglianze, sembra in parte vicino alla volontà di cui parla Lamarck (che Schopenhauer condivide, precisando tuttavia che l'«indimenticabile zoologo» si arresta a un dato punto, non scorgendo che la volontà originaria è metafisica e non fisica) essendo declinata anche nell'accezione di besoin in relazione a un dato ambiente - affine alla necessità di conservarsi, sopravvivere, propria del Wille zum Leben.

33 SCHOPENHAUER, A. WWV I, p. 309.

${ }^{34} \mathrm{Su}$ tale questione, cfr. in particolar modo SCHOPENHAUER, A. WWV I, § 27.

Una possibile conferma postuma: punti di congiunzione tra Schopenhauer e Darwin
} 
resistenza, può considerarsi l'oggettità della volontà nel suo grado più basso, in cui emergono due prerogative fondamentali, il movimento e il conflitto. Ogni corpo celeste ha come natura originaria il movimento, uno spingersi inesauribile che non conosce quiete. Costante è la tensione tra forza centripeta e forza centrifuga, propria del sistema dell'universo. Come il pianeta che, pur dipendente dal corpo centrale, gli oppone resistenza, così le forze chimiche nell'organismo si oppongono al galvanismo. Nella natura inorganica si vedono cristalli che si incontrano e disturbano reciprocamente, in modo tale da non poter assumere la loro forma a punta cristallizzata; calamite che impongono al ferro la propria forza magnetica e «idea». Nell'assimilazione organica l'acqua e il carbonio vengono tramutati in linfa vegetale, e la pianta e il pane vengono trasformati in sangue. Nel regno vegetale e animale, il contrasto appare in modo più chiaro. Si pensi alla lotta silenziosa tra le radici di una pianta o a quella tra i fiori per l'impollinazione, ai rami rampicanti che cercano la luce e che non esitano dall'avvilupparsi su rami che in precedenza si sono mossi in quella direzione. Il mondo animale ha come nutrimento il suo stesso mondo, e quello vegetale. I viventi sono insieme prede e predatori, vinti e vincenti: la volontà di vivere «si nutre di se stessa». Alcuni insetti, come gli icneumonidi, depongono le loro uova nel corpo delle larve di altri insetti, i quali vengono divorati dall'interno con la schiusa delle uova (stesso esempio che abbiamo constatato riporta anche Darwin). La formica-bulldog, tagliata a metà, dà inizio a una lotta tra la parte della testa e della coda, tra morsi e punture. Con l'uomo la lotta raggiunge il grado di maggiore visibilità. Non soltanto perché una buona parte dell'“umanità" considera la natura come un prodotto per il suo uso e consumo, pensando che sia stata creata per servire i suoi egoismi, ma anche perché forme di sopraffazione esistono tra gli uomini stessi, e all' interno di ciascuno, da un punto di vista organico, inerente alle funzioni vitali; etico, se si sceglie di annichilire l'impulso verso l'esistenza che ciascun organismo è, quindi agendo in contrasto con il proprio fenomeno; e della libertà, in relazione al conflitto dei motivi che spingono a una «decisione della scelta» o a un'altra.

La contesa, sebbene essenziale e ineluttabile, non è preminente. Se prevalesse, la vita non sarebbe possibile.

Difatti, per Darwin la selezione naturale raccoglie o elimina variazioni al fine del perdurare. E per Schopenhauer il sussistere di una qualche armonia, di un co- 
adattamento tra i viventi, deriva dalla natura stessa delle cose che, volendo in tutte parimenti persistere, conduce allo scontro ma anche all'incontro. La vita emerge dunque come un avvicendarsi di correlazione e contrapposizione, in cui talvolta si impone la discordia, talaltra la concordia.

Se per Darwin emblema della conservazione è la selezione naturale, e con essa la selezione sessuale35, per Schopenhauer lo è l'istinto sessuale, paradigma dell'affermazione e continuazione della volontà di vivere:

Anche se questa pulsione [sessuale] è già posta dall'esistenza e dalla natura del corpo, il suo appagamento non è tuttavia il semplice volere dell'esistenza [Wollen des Daseyns], della conservazione del proprio corpo, bensì un volere della voluttà [Wollen der Wollust], quindi affermazione della volontà di vivere in un grado molto superiore [...]. La volontà di vivere non si afferma qui entro i limiti della conservazione del corpo, ma in generale, ovvero oltre l'esistenza [Existenz] dell'individuo, la quale riempie un tempo così breve: la vita in quanto tale viene affermata a un grado più elevato, oltre la morte dell'individuo particolare, per un tempo del tutto indefinito. L'intimo significato dell'atto generativo è dunque l'affermazione della vita tout court e non solo l'affermazione della propria individualità ${ }^{36}$.

È un atto che investe la natura tutta, come Schopenhauer descrive ampiamente

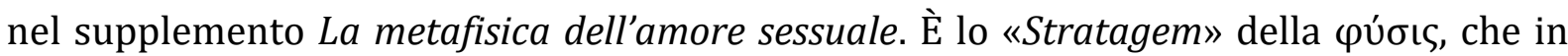
tal maniera persegue il suo fine, il perdurare. È spinta originaria che riguarda ogni vivente, ragion per cui, secondo il filosofo, Eros è stato designato come il padre di tutte le cose.

Esposti questi elementi fondamentali, quello conflittuale e quello conservatore, che ambedue Schopenhauer e Darwin riscontrano nella natura, seppure con tonalità dissimili, ricongiungiamoci alla questione cardinale: il contrapporsi alla spiegazione creazionista, interpretando il «mistero dei misteri» attraverso l'idea di discendenza, sulle prerogative della quale il filosofo e il naturalista per lo più divergono, non spiegandola in maniera simile. Eppure, nonostante le discordanze, quelle che a nostro avviso sono alcune conseguenze del rifiuto della spiegazione teologica sono pressoché le stesse, sintetizzabili nell'espressione usata da entrambi «Natura non facit saltus»; intendendo sia balzi organici, mancanti perché non sono esistiti atti separati di creazione, sia

35 La prima si rapporta al vivente e al suo circostante, la seconda invece concerne essenzialmente la «riuscita di individui rispetto ad altri dello stesso sesso». A riguardo, cfr. DARWIN C. La scelta sessuale, pp. 179-541. In: DARWIN C. L'origine dell'uomo e la scelta in rapporto col sesso.

${ }^{36}$ SCHOPENHAUER, A. Metafisica dei costumi, pp. 89, 90.

Una possibile conferma postuma: punti di congiunzione tra Schopenhauer e Darwin 
intellettivi ed emotivi; ma non ci pronunciamo oltre, approfondendo la questione a breve.

\section{Unità fisica e metafisica. L'«unica grande rete» organica, intellettiva ed emotiva}

Nel contemplare le cose della natura, non si possono non ammirare le innumerevoli forme di cui è composta, il tripudio di colori di cui è tinta, la sinfonia che abbraccia ronzii appena udibili, canti e latrati, strumenti e strutture diversissimi, pinne, zampe, radici e ali, che si muovono in spazi peculiari e condivisi. Osservando la natura, la prima impressione che probabilmente si riceve è di una sconfinata e inafferrabile diversità. Ma immediatamente dopo si potrebbe intuire quanto tale diversità sottenda un'identità. Si pensi che nelle figure più strabilianti e disuguali si presentano gli stessi organi di senso, si svolgono medesime funzioni, si provano comuni emozioni.

Al cospetto di queste somiglianze, l'uomo si è adoperato per comprenderle. Un esempio di tale tentativo è il sistema naturale.

È illuminante a riguardo una descrizione darwiniana, che riportiamo interamente per la sua incisività formale e argomentativa:

I naturalisti cercano di sistemare le specie, i generi e le famiglie di ogni classe secondo quello che viene chiamato sistema naturale. Ma che cosa si intende con questa espressione? Alcuni autori considerano il sistema semplicemente come uno schema per organizzare insieme gli esseri viventi che si somigliano di più e separare quelli che si somigliano meno. Oppure come un mezzo artificiale per enunciare, nel modo più breve possibile, proposizioni di carattere generale, come per esempio indicare con una singola frase i caratteri comuni a tutti i mammiferi, con un'altra quelli comuni a tutti i carnivori, con un'altra quelli comuni al genere cane e poi, con l'aggiunta di un'altra sola frase, descrivere esaustivamente ogni razza di cane. L'ingegnosità e utilità di questo sistema sono fuori discussione. Molti naturalisti, però, pensano che per sistema naturale s'intenda qualche cosa di più: essi credono, cioè, che il sistema riveli il piano del Creatore. Tuttavia, a meno che non si precisi se con il piano del Creatore s'intenda un ordine nel tempo oppure nello spazio, o che cos'altro, mi sembra che con ciò non si aggiunga nulla alla nostra conoscenza. Espressioni come quella famosa di Linneo, che spesso troviamo in forma più o meno dissimulata, secondo cui i caratteri non fanno il genere ma il genere dà i caratteri, sembrano suggerire che le nostre classificazioni hanno a che fare con qualcosa in più della semplice somiglianza. In effetti io credo che ci sia qualcosa in più: la prossimità nella discendenza - l'unica causa nota della somiglianza tra gli esseri organici - è il legame, nascosto da vari gradi di modificazione, che ci viene parzialmente svelato dalle nostre classificazioni. [...] Tutte le 
precedenti regole, gli strumenti di cui ci possiamo servire e le difficoltà che troviamo nella classificazione possono essere spiegati, se non m'inganno, con la concezione secondo la quale il sistema naturale è fondato sulla discendenza con modificazione; i caratteri che, secondo i naturalisti, mostrano la vera affinità tra due o più specie sono i caratteri ereditati da un progenitore comune e dunque, in questo senso, ogni vera classificazione è genealogica; il legame nascosto che i naturalisti hanno cercato inconsapevolmente è la comunità della discendenza, e non un qualche sconosciuto piano di creazione o l'enunciazione di proposizioni generali o il semplice mettere insieme e separare oggetti più o meno simili ${ }^{37}$.

Cerchiamo di capire in che modo Darwin coniuga il nesso affinità-discendenza comune, illustrando che la somiglianza tra i viventi non è decifrabile mediante espressioni quali piano, disegno o volontà del Creatore, che riaffermano soltanto una realtà, senza dispiegarla.

Dunque, Darwin crede che la disposizione dei gruppi in ogni classe debba essere «genealogica», se vuole essere naturale. - La quantità di differenze nei diversi gruppi, nonostante l'affinità con il progenitore comune, può variare a causa dei diversi gradi di modificazione, e anche tale quantità è ordinata in generi, famiglie, sezioni o ordini. Supponendo che le lettere da A a L costituiscono generi affini vissuti durante il periodo silurano, e che sono discesi da una specie di un periodo antecedente, «alcune specie di tre di questi generi (A, F, I) hanno trasmesso discendenti modificati fino a oggi, rappresentati da quindici generi (da a14 a z14) sulla linea orizzontale più alta. Ora, tutti questi discendenti modificati di una singola specie sono rappresentati come collegati dallo stesso grado di parentela; metaforicamente potremmo chiamarli tutti cugini dello stesso, milionesimo grado, eppure differiscono ampiamente e in gradi diversi l'uno dall'altro ${ }^{38 . » ~ L e ~ f o r m e ~ d e r i v a t e ~ d a ~ A ~ c o s t i t u i s c o n o ~ u n ~ o r d i n e ~ d i v e r s o ~ d a l l e ~ f o r m e ~ d e r i v a t e ~}$ da I, così come le forme discese da A non possono costituire lo stesso genere del progenitore A, né quelle discese da I far parte dello stesso genere del progenitore I. Invece, è possibile supporre che il genere esistente F14 si sia modificato poco e che quindi possa essere classificato insieme al genere progenitore F. Così, la quantità o il valore delle differenze tra esseri organici, tutti imparentati tra loro, è diventato molto vario. Ciò nonostante, i discendenti modificati di A avranno ereditato tratti comuni dal loro comune progenitore, parimenti i discendenti di I, e così per ogni ramo subordinato

37 DARWIN, C. L'origine, pp. 436, 442.

${ }^{38}$ Ivi, p. 443.

Una possibile conferma postuma: punti di congiunzione tra Schopenhauer e Darwin 
di discendenti, in ogni periodo successivo. Presumendo che alcuni dei discendenti di A e di I si siano modificati, a tal punto da aver perso ogni traccia della loro ascendenza, allora i posti occupati da essi in una classificazione naturale saranno all'incirca perduti. Ammettendo che tutti i discendenti del genere F, lungo la linea di discendenza, si siano modificati poco, allora formeranno ancora un solo genere. Quindi, per tali ragioni, Darwin asserisce che «il sistema naturale è genealogico nella sua disposizione come un pedigree; ma i gradi di modificazione a cui i diversi gruppi sono stati soggetti devono essere espressi raggruppandoli sotto quelli che chiamiamo generi, sottofamiglie, famiglie, sezioni, ordini e classi diverse ${ }^{39} . »$

Anche per classificare le varietà si seguono gli stessi criteri utilizzati per raggruppare le specie: caratteri costanti, l'individuazione di organi rudimentali o atrofizzati, distribuzione geografica, somiglianze tra le parti, estinzione.

Quindi, ricapitolando, se la discendenza è stata usata per classificare sia gli individui della stessa specie che le varietà, Darwin domanda retoricamente:

Non si può forse ritenere che questo stesso elemento della discendenza sia stato utilizzato inconsapevolmente per raggruppare le specie nei generi e i generi nei gruppi più alti, anche se in questi casi il grado della modificazione è stato maggiore e ha richiesto più tempo per completarsi? Io credo che la discendenza sia stata usata proprio in questo modo, e solo così posso comprendere le diverse regole e istruzioni seguite dai nostri migliori sistematici ${ }^{40}$.

Continuando, Darwin sottolinea che per ricostruire la discendenza comune, non disponendo di alberi genealogici scritti, occorre basarsi su «ogni tipo di somiglianza». Bisogna anche tener presente che l'affinità dei caratteri è soggetta alle leggi della loro trasmissibilità, ed è complicata dal principio della moltiplicazione e graduale divergenza dei caratteri, dovute a più fattori: «il progenitore comune di un'intera famiglia di specie, ora divisa dall'estinzione in gruppi e sottogruppi distinti, avrà infatti trasmesso a tutti alcuni suoi caratteri, modificati in vari modi e misure; e le diverse specie saranno perciò collegate le une alle altre da linee di affinità tortuose e di varia lunghezza che risalgono attraverso molti predecessori ${ }^{41}$.»

Ebbene, le affinità sono rivelate in particolar modo dalla morfologia e

\footnotetext{
${ }^{39}$ Ivi, p. 444.

40 Ivi, p. 448.

41 Ivi, pp. 453-454.

Una possibile conferma postuma: punti di congiunzione tra Schopenhauer e Darwin
} 
dall'embriologia. La prima costituisce per Darwin il settore più interessante della storia naturale, essendone l'«anima stessa ${ }^{42} »$, nella misura in cui coglie le somiglianze, in un'accezione molto vasta, tra tutti gli esseri.

La mano dell'uomo, quella della talpa, la zampa del cavallo, la pinna della focena e l'ala del pipistrello rimandano tutte, osserva Darwin, a uno stesso modello. Hanno le stesse ossa nelle stesse posizioni. Cambiano le forme e le dimensioni, ma l'ordine che le collega è il medesimo ${ }^{43}$. Difatti, ad esempio, le ossa di un arto possono essere accorciate o allargate, restare avvolte in spesse membrane e quindi fungere da pinne, diventare ali:

Ma in tutte queste notevoli modificazioni non ci sarà la tendenza a modificare la struttura complessiva delle ossa o la connessione relativa delle diverse parti. Se supponiamo che l'antico progenitore - potemmo chiamarlo l'archetipo dei mammiferi - possedesse gli arti costruiti sul modello generale attuale, a qualunque scopo gli servissero, comprendiamo immediatamente il semplice significato della costruzione omologa degli arti in tutta la classe ${ }^{44}$.

Qualcosa di analogo si presenta nella formazione della bocca degli insetti: molto diverse sono la lunghissima proboscide a spirale della farfalla sfinge, la spirale ripiegata di un'ape o di una cimice e le grandi mandibole di un coleottero. Eppure, queste forme distinte rimandano a un'uguale struttura di riferimento, che ha subito svariate modificazioni: un labbro superiore, mandibole e due paia di mascelle comuni. Lo stesso processo riguarda la configurazione di un fiore e di qualsiasi altro vivente, il cui corpo, nella sua specificità, declina un che di comune.

Un altro studio che rivela notevoli affinità tra gli animali è l'embriologia, nella misura in cui alcune parti corporee, nello stadio embrionale, sono identiche, invece con la crescita e in età adulta differiscono notevolmente.

A sostegno di tale rassomiglianza, Darwin racconta che Agassiz, dopo aver

\footnotetext{
42 Ivi, p. 457. Anche per il filosofo di Danzica la morfologia è di grande rilevanza: per Schopenhauer le forme dei viventi sono profondamente rivelative, raffigurando quell'identico tendere verso la vita concorde a determinate e diverse circostanze, quindi necessità di esistere in un dato spazio, con abitanti ben individuati, in precise condizioni: «Le varie forme animali nelle quali la volontà di vivere si presenta hanno tra loro lo stesso rapporto che ha lo stesso pensiero espresso in lingue diverse e conformemente allo spirito di ognuna di esse [e le varie specie di un genere si possono considerare come un certo numero di variazioni sullo stesso tema]» (SCHOPENHAUER, A. P II, p. 233). Primariamente, la conformazione del corpo umano (con i suoi moti e le sue azioni) rappresenta quel «passaggio segreto» per approssimarsi alla cosa in sé, quindi per carpire che l'intero mondo corporeo è manifestazione della volontà.

${ }^{43}$ È il cosiddetto «elemento anatomico» sulla cui importanza ha insistito molto Geoffrey St. Hilaire, a cui non poteva non fare riferimento anche Schopenhauer, riscontrando anch'egli l'«unità di tipo», seppur conducendola in ultima istanza a un che di metafisico. Infra, p. 25.

44 Ibidem.

Una possibile conferma postuma: punti di congiunzione tra Schopenhauer e Darwin
} 
dimenticato di etichettare un embrione di alcuni vertebrati, non era in grado di dire se si trattasse di un mammifero, di un rettile o di un uccello. Anche le larve di falene, mosche e coleotteri si somigliano tantissimo. Talvolta, tracce di similarità tra embrioni persistono fino a uno stadio avanzato: uccelli dello stesso genere si somigliano anche nel primo e secondo piumaggio; tra i gatti molte specie sono striate o macchiate come altri felini; alcune piante come l'Ulex e l'Acaceas phyllodineous sono pennate come le foglie ordinarie delle leguminose.

Quindi, come si spiegano l'incredibile somiglianza tra embrioni e la notevole divergenza da adulti? La discendenza comune con modificazione può chiarire anche tale accadere. In primo luogo occorre dire che le variazioni, marcate, non appaiono fin dai primissimi stadi dell'embrione. Inoltre, bisogna distinguere il periodo della vita in cui una variazione è stata causata da quello in cui si mostra compiutamente, poiché la causa del mutamento può risalire anche a molto tempo prima della formazione embrionale, tuttavia apparire non in tenera età; inoltre, spesso accade che le variazione tendono a presentarsi nella prole nell'età i cui si sono manifestate nei genitori. Per giunta, l'uso o il disuso di un organo influenza l'animale maturo, non il piccolo, e gli effetti legati all'esercizio prolungato o al suo contrario saranno ereditati a un'età corrispondente a quella dei genitori. Per questo motivo gli embrioni, fino a determinati stadi, non si distinguono nettamente gli uni dagli altri.

Ebbene, la somiglianza di fondo si spiega con l'esistenza di un comune progenitore, le differenze nei confronti del quale, legate alle leggi di trasmissibilità dei caratteri, si manifestano in un secondo tempo ${ }^{45}$. Vengono selezionate, accumulate o scartate dopo la nascita, presentandosi non negli embrioni ma nella loro conformazione matura.

Ricapitolando,

se due gruppi di animali, per quanto oggi diversi l'uno dall'altro per struttura e abitudini, passano attraverso stadi embrionali identici o simili, possiamo star certi che sono entrambi discesi da progenitori identici o molto simili, e sono quindi in tale misura correlati. Pertanto una struttura comune nell'embrione rivela la comunità della discendenza; e la rivelerà indipendentemente da quanto la struttura dell'adulto possa essere modificata e alterata ${ }^{46}$.

45 Cfr. DARWIN, C. L'origine, pp. 462-474.

46 Ivi, p. 473.

Una possibile conferma postuma: punti di congiunzione tra Schopenhauer e Darwin 
L'embrione dunque può considerarsi un'«immagine più o meno sbiadita» della comune forma progenitrice di ogni classe. Per questo la sua figura è altamente significativa e conseguentemente il suo studio di grande rilevanza ${ }^{47}$. Affinità embriologiche si affiancano ad affinità morfologiche di età mature, evocando nel loro complesso una comune appartenenza e provenienza, non uranica. E non sono le uniche similarità che intrecciano tutti i viventi. La rete che li lega gli uni agli altri è anche genetica e chimica. Tutte queste somiglianze non sono riconducibili a una volontà divina, lasciando essa inspiegate le meraviglie del cosmo, solo ribadite e ricondotte a un asserto. La domanda intorno al perché di siffatte disposizioni, relazioni, forme, non riceve risposta. Di contro, la teoria della discendenza comune con modificazione, insieme al principio della selezione naturale, tenta ed è in grado di porgere alcune risposte. È inoltre fondamentale riconoscere la lentezza con cui la selezione opera, apportando lievi modificazioni successive. Quindi dal suo agire derivano gradazioni innumerevoli, ramificazioni molteplici innestate su comuni radici, oramai estinte. Gradazioni che riguardano non soltanto le forme, i colori, gli organi, ma anche gli istinti. Pertanto, il principio secondo cui «Natura non facit saltus» abbraccia l'organismo nella sua totalità, significando che non si danno balzi né di natura strettamente corporea, per cui ogni animale sarebbe il risultato di irrelati atti di creazione, né salti di natura istintivo-

47 Oltre alla morfologia e all'embriologia, per Darwin, un altro studio rivelativo di una comune discendenza concerne gli organi rudimentali, atrofizzati o abortiti, quindi quegli organi sostanzialmente inutili, inutilizzabili, che vanno dalle mammelle nei mammiferi maschi alla presenza di denti allo stato fetale delle balene che da adulte non hanno nemmeno un dente, dalle ali atrofizzate agli occhi coperti da palpebre fisse: «Secondo la mia concezione della discendenza con modificazione l'origine degli organi rudimentali è semplice. [...] Io credo che l'agente principale sia stato il disuso, che ha portato in generazioni successive alla riduzione graduale di vari organi fino al loro diventare rudimentali, come nel caso degli occhi degli animali che abitano nelle caverne buie e le ali degli uccelli che abitano le isole oceaniche, che raramente sono stati forzati a prendere il volo e, alla fine, hanno perso la capacità di volare. [...] Qualsiasi cambiamento di funzione che possa essere realizzato per passaggi impercettibilmente piccoli rientra nei poteri della selezione naturale; così un organo reso inutile o dannoso per uno scopo in condizioni di vita mutate può essere facilmente modificato e utilizzato per un altro scopo. [...] In qualunque periodo della vita il disuso o la selezione riducano un organo - e generalmente sarà quando l'essere ha raggiunto la maturità e i suoi pieni poteri d'azione - il principio dell'eredità a età corrispondenti riprodurrà l'organo nella sua condizione ridotta alla stessa età e, di conseguenza, raramente lo influenzerà o ridurrà nell'embrione. Così possiamo comprendere le dimensioni relativamente maggiori che gli organi rudimentali hanno nell'embrione e le dimensioni relativamente ridotte che presentano invece nell'adulto. [...] Gli organi rudimentali possono essere paragonati alle lettere di una parola che, pur essendo conservate nell'ortografia, sono inutili nella pronuncia e ci servono come un indizio quando cerchiamo da dove deriva. Secondo la concezione delle discendenza con modificazione possiamo concludere che l'esistenza di organi in una condizione rudimentale, imperfetta e inutile, o addirittura abortiti, anziché costituire una strana difficoltà - com'è certamente per la dottrina comune della creazione - poteva addirittura essere prevista e può essere spiegata con le leggi dell'eredità.» Ivi, p. 479.

Una possibile conferma postuma: punti di congiunzione tra Schopenhauer e Darwin 
intellettiva ed emotiva, passando da animali macchina ad animali coscienti.

Ammettendo ciò, «se le specie sono discese da altre specie attraverso gradazioni impercettibili, perché non vediamo ovunque un numero senza fine di forme di transizione? Perché la natura non si trova in uno stato di confusione, e le specie che vediamo sono invece ben definite $?^{48\rangle}$

I motivi per cui non contempliamo «rive intricate» di forme intermedie sono vari, ma possiamo ricondurli a due cause fondamentali: il procedere stesso della selezione naturale è nel contempo accumulazione ed estinzione (prima causa). Una nuova forma tenderà a prendere il posto dei suoi progenitori meno perfezionati o di altre forme meno avvantaggiate rispetto alle circostanze, con cui entra in competizione. Vale a dire che le varietà intermedie saranno soppiantate dallo stesso processo di formazione della nuova varietà. Posto questo, rimarrebbe ancora da obiettare ciò: «ma poiché secondo questa teoria devono essere esistite innumerevoli forme di transizione, come mai non le troviamo sepolte nella crosta terrestre in un numero pressoché infinito?» La documentazione fossile è meno completa di quanto comunemente si suppone (seconda causa). Tale parzialità è dovuta primariamente al fatto che gli esseri organici non vivono nelle profondità del mare, in cui poter persistere, e i loro resti vengono conservati per un arco temporale esteso solo quando si trovano in masse di sedimenti ben resistenti a un enorme livello di degrado. Quindi, annota Darwin, ci sono inevitabili lacune nella storia geologica: «la crosta della terra è un grande museo, ma le collezioni naturali sono state realizzate a intervalli di tempo immensamente lunghi ${ }^{49}{ }^{»}$. Nondimeno, egli non dubita di ciò a cui i fatti, seppur esigui, sembrano alludere.

Quindi, alla luce di quanto esaminato, Darwin ritiene che la discendenza con modificazione abbracci tutti i membri della stessa classe, credendo che gli animali siano discesi da non più di quattro o cinque progenitori e le piante pressoché dallo stesso numero. L'analogia addirittura lo «spingerebbe oltre», a credere che tutti gli animali e le piante sono discesi da un solo progenitore, ma talvolta essa può costituire una «guida ingannevole».

Come che sia, si può contemplare che «tutte le cose viventi hanno molto in comune»: nelle strutture basilari, nelle funzioni, nella composizione chimica, nelle

\footnotetext{
48 Ivi, p. 188.

${ }^{49}$ Ivi, p. 189.

Una possibile conferma postuma: punti di congiunzione tra Schopenhauer e Darwin
} 
vescicole germinali, nella struttura cellulare, nelle leggi della crescita e della riproduzione. Perciò, «[...] probabilmente, tutti gli esseri organici mai esistiti su questa terra sono discesi da una qualche forma primordiale, nella quale la vita venne infusa per la prima volta ${ }^{50}$ ». Ancor prima di tale forma, Schopenhauer pone l'«animale originario», l'Urthier, la volontà informe, onniforme. Ciò che soggiace ai progenitori comuni. Ciò che perdura. Il metafisico nel fisico. E ancor prima non è dato risalire.

Dobbiamo arrestarci di fronte a presunte strutture progenitrici, non avendo gli «archetipi» in forma fossile, ma potendo presagirne i tratti mediante i fenomeni che da essi sono discesi. Così come, analogamente, tentiamo di descrivere la volontà come Ding an sich attraverso le sue oggettivazioni, i suoi rivestimenti più tenui.

Anche per Schopenhauer le cose della natura sono affini. Manifestano l'unità e identità nella diversità. I corpi più dissimili sono oggettivazione di quello stesso volere che è anelito alla vita e alla sua continuazione, per questo tentativo di adattamento e riproduzione (o, in un senso più esteso e generale, tensione alla conservazione, che è riproduzione, ri-posizione dello stesso).

La gravità, la circolazione sanguigna, la ciclicità della pianta, l'istinto materno sono tutti paradigmi differenti che indicano la medesima essenza: il movimento perenne che la vita è e vuole continuare ad essere, conservandosi. Inoltre, questo impulso a persistere, conato fondamentale del volere, si configura nella concordanza tra il corpo e il suo modo di vivere, e tra le sue parti interne. Altrimenti detto, la necessità di dover sopravvivere in determinate circostanze ha condotto e conduce a una formazione a esse adeguate.

Muoviamo ora da una considerazione chiave circa le somiglianze morfologiche, emblematica sia per quello che suggerisce sia per i punti di contatto tra il pensiero schopenhaueriano e darwiniano:

Si comprende bene, per esempio, perché il cranio dell'uomo sia composto di otto ossa, cioè affinché queste, per mezzo delle fontanelle, possano stringersi insieme al momento della nascita; ma perché il pulcino che rompe il suo uovo, debba avere lo stesso numero di ossa craniche non si capisce. Dobbiamo quindi ammettere che questo elemento anatomico riposi in parte sull'unità e identità della volontà di vivere in genere, e in parte sul fatto che le forme originarie degli animali sono derivate l'una dall'altra (Parerga, vol. 2, § 91) e quindi il tipo di

${ }^{50} I v i$, p. 509.

Una possibile conferma postuma: punti di congiunzione tra Schopenhauer e Darwin 
base dell'intera stirpe è stato mantenuto ${ }^{51}$.

Ebbene, la morfologia rivela al filosofo ciò che accomuna i viventi: e dei tipi di base, da cui sarebbero derivate forme animali (constatazione non molto dissimile da quella darwiniana, che pone a fondamento di tutte le affinità progenitori comuni); e l'identità e unità della volontà di vivere, che chiaramente Darwin non pone, ma che molto vagamente, solo per alcuni tratti, potremmo rintracciare nella selezione naturale.

I concetti nodali per comprendere l'idea schopenhaueriana di discendenza sono la generatio aequivoca o spontanea e la generatio in utero heterogeneo.

- Primariamente, occorre tener presente la distinzione tra cosa in sé, idea e fenomeno ${ }^{52}$, nella misura in cui «ogni cosa fisica è d'altra parte insieme una cosa metafisica53». La volontà, quale Ding an sich, quale fondamento originario di tutte le cose, Urgrund, è inconoscibile, poiché estranea alla rappresentazione, «oggetto per un soggetto», e alla sua forma, il principio di ragion sufficiente. In quanto per sé sfugge a uno sguardo che possa contemplarla. Motivo per cui possiamo approssimarci ad essa soltanto mediante il suo «fenomeno più immediato», il corpo. È indipendente dallo spazio e dal tempo. È singolare, nella misura in cui è al di là di essi. È ingenerata e imperitura. È identica e intera ovunque. La sua essenza non muta negli innumerevoli e svariati fenomeni naturali, cambiano soltanto le qualità secondarie che la adornano. È causa di tutte le cose ma non è causata, o almeno non ci è dato conoscere il suo «donde» e la sua «fatalità». Dal conflitto della volontà con se stessa, dovuto al tendere onnidirezionale e perpetuo della sua stessa natura, derivano le idee, «gradi di oggettivazione immediati e adeguati» della cosa in sé, della quale condividono gli stessi attributi. Mediante la «legge naturale» le idee si relazionano con le forme dei fenomeni e così si perviene alle oggettivazioni «mediate», i corpi, entia realissima. Soggetti alle leggi

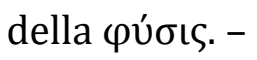

Orbene, per il filosofo, se la nascita di endozoi, epizoi e infusori è spiegabile

51 SCHOPENHAUER, A. N, p. 118. Anche Darwin, a sostegno di una certa unità nella natura, avrebbe riportato un esempio pressoché uguale a quello schopenhaueriano: «Perché mai il cervello dovrebbe essere racchiuso in una scatola composta di pezzi d'ossa tanto numerosi e dalla forma così singolare? Come ha osservato Owen, il vantaggio che deriva dalla cedevolezza dei pezzi separati nell'atto del parto dei mammiferi non spiegherà in alcun modo la stessa costruzione del cranio degli uccelli.» (DARWIN, C. L'origine, p. 460). Per giunta, circa la morfologia, entrambi si rifanno soprattutto agli studi di Richard Owen, Geoffrey Saint Hilaire, e Georges Cuvier, come attestano non pochi riferimenti a tali studiosi.

52 Cfr. SCHOPENHAUER, A. WWV I, § 23, § 24, § 25, § 26, § 27.

53 SCHOPENHAUER, A. WWV II, p. 1339.

Una possibile conferma postuma: punti di congiunzione tra Schopenhauer e Darwin 
equivocamente, quindi attraverso una genesi dal limo, dalla viscosità del mare, da una massa organica in putrefazione, da un corpo vegetale morto, non è in tal modo spiegabile la nascita dell'uomo e degli altri animali.

Questa la loro genesi, stando alla descrizione di Schopenhauer, discutibile e farraginosa - soprattutto alla luce de L'origine delle specie. La riportiamo quasi interamente anche per dare un'idea dell'insolita non chiarezza espositiva54:

[la nascita dell'uomo e di altri animali] può essere pensata soltanto come generatio in utero heterogeneo, di conseguenza in modo tale che dall'utero - o piuttosto dall'uovo di una coppia animale particolarmente favorita, dopo che la forza vitale della sua specie, ostacolata da un qualsiasi impedimento, si sia accumulata e accresciuta in modo anormale proprio in questa coppia [...] - sarebbe nata eccezionalmente non più la forma eguale ma quella affine a quella coppia, tuttavia a un grado superiore; sicché tale coppia, questa volta, non avrebbe generato soltanto un individuo, ma una specie. Processi di questo genere hanno potuto naturalmente verificarsi solo dopo che gli animali infimi si erano portati alla luce, mediante la solita generazione equivoca, dalla putredine organica o dal tessuto cellulare di piante viventi, quasi primi messaggeri e quartiermastri delle specie animali avvenire. Un tal processo dev'essere avvenuto dopo ciascuna di quelle grandi rivoluzioni terrestri, che almeno tre volte hanno completamente distrutto ogni forma di vita sul pianeta, di modo che questa doveva riaccendersi, presentandosi poi ogni volta in forme più perfette, cioè più vicine alla fauna attuale. Ma solo nella serie animale, subentrata dopo l'ultima grande catastrofe della superficie terrestre, quel processo si è intensificato fino alla nascita del genere umano, dopo essere giunto, già dopo la penultima catastrofe, fino alla scimmia. [I batraci conducono davanti ai nostri occhi una vita di pesci prima di assumere la loro forma più compiuta e, secondo un'osservazione oggi abbastanza

\footnotetext{
54 Molto pertinente sulla prosa schopenhaueriana è un'osservazione di Colli: «La sua esposizione filosofica non teme confronti, negli ultimi secoli. Profonda, rigorosa, limpida, spiritosa, varia, brillante. Il suo stile non solo è raffinato e ampio, equilibrato e concreto, ma riscalda, consola nella solitudine, è intimo, premuroso verso chi vuol capire. E l'intelletto è lucido, i concetti si riannodano sempre all'intuizione, la ragione è sana. Le stesse parole hanno ogni volta lo stesso significato, le definizioni sono chiare, il ragionamento persuasivo. E la coerenza è la perla dell'edificio. Che si può chiedere di più a un filosofo?» (COLLI, G. prefazione a Arthur Schopenhauer, La quadruplice radice del principio di ragion sufficiente. In: COLLI, G. Per una enciclopedia di autori classici, pp. 118-119). Molto calzante è anche l'analisi di Mann, che lega intimamente lo stile e il contenuto della filosofia schopenhaueriana: «La filosofia di Arthur Schopenhauer è stata sempre intesa come eminentemente artistica, la filosofia dell'arte par excellence. E non perché in così alto grado, per una parte così cospicua, essa è una filosofia dell'arte - l'estetica occupa infatti un quarto di tutta l'opera -; e nemmeno perché la sua composizione è così perfettamente chiara, trasparente, compatta, il suo linguaggio vigoroso, elegante, preciso, passionale e arguto, di una purezza classica e di una grandiosa e serena severità stilistica, quali mai si erano viste fino ad allora nella filosofia tedesca. Tutto ciò non è che "apparenza", fenomeno, forma di bellezza innata e necessaria per esprimere la sostanza, l'intima natura del suo pensiero; natura piena di tensione, fortemente affettiva, sospesa tra violenti contrasti, tra istinto e spirito, passione e redenzione, una natura così dinamicamente artistica da non potersi esprimere che in forme di bellezza, come una creazione di verità personale, la cui forza persuasiva si fonda tutta su un'esperienza vissuta e sofferta» (MANN, T. Schopenhauer. In: MANN, T. Nobiltà dello spirito e altri saggi, pp. 1237-1238).

Una possibile conferma postuma: punti di congiunzione tra Schopenhauer e Darwin
} 
universalmente riconosciuta, ogni feto percorre successivamente le forme delle classi che stanno al di sotto della sua specie, finché giunge alla propria. Ma, allora, ogni specie nuova e superiore non potrebbe essere nata proprio perché questa intensificazione della forma del feto una volta ha superato di un gradino la forma della madre che lo portava? Questa è l'unica escogitabile maniera razionale, cioè ragionevolmente pensabile, con cui può essere avvenuta la nascita delle specie 55 .

Inoltre, tale intensificazione non va pensata come procedente su un'unica linea, ma su molteplici: dall'uovo di pesce sarebbe nato un ofidio, e da questo un sauro; contemporaneamente, altrove, da quello di un altro pesce un batrace e poi dall'uovo di quest'ultimo un chelone; un cetaceo avrebbe generato una foca e una foca un tricheco; uno struzzo un mammifero più grande e così via.

Ci sembra superfluo commentare quanto sia lampante la divergenza con la teoria darwiniana. La differenza emerge chiaramente, nella misura in cui, per quanto si possa accettare l'idea di un'intensificazione, nei termini di mutamento-miglioramento, non si può che respingere l'ipotesi di un'intensificazione embrionale, dato che l'embrione è ben lungi dall' essere, al suo inizio, qualcosa di fortemente specifico. È stato poc'anzi constatato con Darwin che alcuni cambiamenti non sopravvengono in quella fase vitale, ma appaiono nello stadio avanzato dell'embrione. Anzi, molti si presentano dopo la nascita, talvolta pressappoco nello stesso periodo in cui le modificazioni si sono presentate nei genitori. Il movimento è inverso rispetto a quello descritto da Schopenhauer.

Ad ogni modo, la caratteristica che sembrerebbe distanziare decisamente i due pensatori riguarda il ritenere, da parte di Schopenhauer, che tutti questi nuovi gradini siano stati «subito determinati e netti», «ognuno dei quali produceva una specie fissa e permanente, non però per passaggi graduali ${ }^{56}{ }_{\Downarrow}$.

Al contrario, per Darwin la lentezza e la gradazione costituiscono due elementi fondamentali della discendenza comune con modificazione.

En passant, ricordiamo che la concezione schopenhaueriana della generatio è corroborata dalle ricerche, note anche a Darwin, dell'anonimo autore delle Vestiges of

\footnotetext{
55 SCHOPENHAUER, A. P II, pp. 200-201.

56 Ivi, p. 202. Continuando, «Dunque, non secondo l'analogia di una nota che salga poco a poco dall'ottava più bassa alla più alta, ed è quindi stridula, bensì secondo l'analogia di una scala che sale in determinate intervalli. Non ci nascondiamo che dovremmo immaginarci i primi uomini come generati in Asia dal pongo [il cui piccolo si chiama orango] e in Africa dallo scimpanzé, sia pure non come scimmie ma subito come uomini» (Ibidem).

Una possibile conferma postuma: punti di congiunzione tra Schopenhauer e Darwin
} 
natural history of Creation ${ }^{57}$. Si tratta di un elemento che ribadisce il costante dialogo del filosofo con le scienze naturali, iniziato durante gli anni universitari e mai interrotto, rintracciabile nei suoi scritti, editi e inediti, condensato in Sulla volontà nella natura, e presente fino ai Parerga.

Ora, accennata la divergenza circa il modo di intendere l'origine della vita, volgiamoci a indicare alcune somiglianze strettamente connesse al comune rifiuto dell'interpretazione teologica.

Esclusa l'ipotesi degli atti separati di creazione, ammessa una comune discendenza, di stampo fisico per Darwin e, prima di tutto, metafisico per Schopenhauer, entrambi osservano che le affinità tra i viventi non sono soltanto di natura morfologica, embriologica, cellulare, chimica; in una parola organica stricto sensu, ma riguardano anche altre parti del corpo: le affezioni, le idee, le volizioni, i pensieri.

Il corallo della vita si colora dunque di comuni tonalità intellettive ed emotive. Innumerevoli e varie sono le forme strutturali dei viventi e altrettanto varie sono le forme del sentire. In questa eterogeneità sorprendente si ravvisa l'omogeneità: come gli esseri sono imparentati tra loro, discendendo da comuni progenitori, dunque possedendo modelli organici affini, così sono legati gli uni agli altri in un'«unica rete» del patire, condividendo affini sentimenti e modi di intelligere.

Insomma, l'unità del principio e l'unità di tipo, in termini schopenhaueriani, e l'unità di tipo, in termini darwiniani, spiega e dispiega il comune nel diverso, sia esso

\footnotetext{
${ }^{57}$ Utile, per un quadro più chiaro della ricezione delle Vestiges sia da parte di Schopenhauer che di Darwin, quanto delucida Volpicelli: «Le Vestiges of the Natural History of Creation, pubblicate a Londra nel 1844, suscitarono una violenta reazione negli ambienti religiosi e scientifici. Adam Sedgwick, ad esempio, in una severissima recensione apparsa nel 1845 in 'The Edinburgh Review', la definì un'opera piena delle 'peggiori assurdità di un materialismo lugubre e irrazionale' e Hugh Miller, nel 1847, ne confutò le tesi fondamentali nei Foot-Prints of the Creator. Or, the Asterolepis of Stromness. Nonostante le aspre critiche, l'opera ebbe enorme successo e larghissima diffusione, giungendo nel breve spazio di sette mesi alla quarta ristampa, fino a superare, con l'undicesima edizione, nel 1860, le ventimila copie vendute. 'È mia opinione - così scriveva Charles Darwin a proposito delle Vestiges - che questa opera abbia reso un eccellente servizio in questo paese con il richiamare l'attenzione sul tema, col rimuovere pregiudizi e preparare così il terreno per la ricezione di vedute analoghe.' (cfr. Charles Darwin, An Historical sketch of the progress of opinion on the origin of species, in ID., On the Origins of Species by Means of Natural Selection, or the Preservation of Favoured Races in the Struggle of Life, Sixth edition, London, J. Murray, 1882, p. XVII). Numerose furono le congetture relative alla paternità di quell'opera, attribuita tra gli altri a Charles Lyll e al principe Alberto, marito della regina Vittoria. Solo nel 1884, nella introduzione alla dodicesima edizione, Alexander Ireland, svelando il segreto di cui era depositario, la attribuì definitivamente a Robert Chambers (1802-1871). Cfr., in particolare, Milton Millhauser, Just before Darwin. Robert Chambers and 'Vestiges', Middletown, Conn., 1959. Oltre alle Vestiges, Schopenhauer lesse anche le successive Explanations: a Sequel to 'Vestiges of the Natural History of Creation' (1845). Entrambe le opere, da lui attribuite a Lindley Kemp, autore di The phases of Matter (1855) si trovano nella sua biblioteca, come documentato in HN V, [886], [887]. Cfr. Wilhelm Brauns, Schopenhauer und die 'Vestiges', in 40. Jb. (1959), pp. 53-55», VOLPICELLI, I. A. Schopenhauer, la natura vivente e le sue forme, p. 40. 
organico, cognitivo o emotivo. Da ciò segue anche che le differenze, che pur sussistono, sono graduali, moventi lievemente e lentamente dalla semplicità alla complessità, e non nette, passanti ad esempio da un animale privo di intelletto a uno provvisto, da un automa a un essere senziente. Ogni vivente possiede inimitabili forme corporee, peculiari forme di intelligenza e tonalità emotive, e in queste manifestazioni afferma congiuntamente la sua propria specificità, il diverso, e un che di condiviso, l'identico. Rivela il simile nel dissimile, e viceversa, e tale intreccio dialettico non conosce balzi, né organici, né cognitivi o affettivi. Il «Natura non facit saltus» si riferisce agli individui nella loro interezza. È un residuo creazionista, e cartesiano, ma non solo, credere che gli animali non umani siano dotati di istinti e gli animali umani di intelletto, creando uno iato tra i due termini, un'antitesi inconciliabile, un vuoto che, stando alla teoria della discendenza, non sussiste. Questo non implica rinnegare le differenze, ma significa non marcarle oltre la loro stessa natura.

Dunque, per Darwin e Schopenhauer non soltanto tutti gli animali sono esseri intelligenti, attributo dalla definizione plurale, ma gli istinti che possiedono, e che si diversificano dalle capacità intellettive, non sono qualcosa di completamente slegato da queste. I due termini non costituiscono un dualismo oppositivo o due linee parallele, sono prerogative che si intersecano.

Schopenhauer ritiene che l'istinto, nel suo complesso, sia un'attività in cui la conoscenza ha un ruolo di accompagnamento e non di guida. Altrimenti detto, essa non è l'elemento predominante.

I punti di vista sulla questione, più diffusi all'epoca, erano riconducibili a due considerazioni antitetiche: il ritenere l'animale un vivente dotato di intelligenza, agente finalisticamente; il reputarlo pressappoco una macchina. Secondo la prima concezione, ammirando le opere di cui gli animali sono capaci, i loro spostamenti e comportamenti, si potrebbe essere indotti a credere che l'intero loro agire sia mosso da rappresentazioni finalistiche, quindi da una riflessione preliminare cui segue l'esecuzione. Viceversa, stando alla seconda, uno sguardo antropocentrico desumerebbe uno svolgimento meccanico, automatico, proiettando la propria cecità sul fare animale ${ }^{58}$.

Schopenhauer non concorda pienamente con nessuna delle due posizioni.

Circa la prima, il giudicare che un certo modo di agire sia il risultato di

${ }^{58}$ Cfr. SCHOPENHAUER, A. Metafisica della natura, pp. 53-54.

Una possibile conferma postuma: punti di congiunzione tra Schopenhauer e Darwin 
ragionamenti connessi al concetto di fine è erroneo nella misura in cui alcuni comportamenti necessiterebbero di un'esperienza precedente, di una conoscenza dell'esistenza e della natura degli oggetti in questione, prerogative che gli animali, in determinate condizioni, non possiedono: «L'uccello di un anno non ha alcuna rappresentazione delle uova per le quali costruisce un nido; il ragno non ne ha della preda in funzione della quale tesse la rete; così il formicaleone, il cervo volante ${ }^{59 . »}$

Circa la seconda posizione, Schopenhauer esprime un maggiore disappunto, non ritenendo affatto che l'animale possa equipararsi a una macchina, non avendo niente in comune con essa. Rispetto a questa concezione, Schopenhauer appura che «[...] dall'ingranaggio della loro organizzazione dovevano risultare necessariamente e del tutto involontariamente, così come le funzioni interne della digestione e simili, anche i movimenti esterni, attraverso i quali gli artefatti vengono realizzati, né più né meno che una macchina per tessere o per filare. Ma una macchina segue il proprio programma in maniera fissa e immutabile, senza deviare da esso qualora le circostanze lo richiedano ${ }^{60 . » ~ A l ~ c o n t r a r i o, ~ l ' a n i m a l e ~ e ̀ ~ i n ~ g r a d o ~ d i ~ m o d i f i c a r e ~ i l ~ p r o p r i o ~ a g i r e, ~ d i ~}$ attendere il tempo opportuno, di servirsi di altri mezzi, di valutare luoghi adatti.

Una macchina ripete ciecamente ciò per cui è stata progettata e azionata. Se per qualche motivo un passaggio viene disturbato, se subentra un guasto, un elemento imprevisto, essa prosegue, come se le circostanze non esistessero. Non può adoperarsi per modificare una condizione inattesa poiché non ha conoscenza, non può agire altrimenti da come è stata programmata. Il suo essere è costrizione.

L'animale, se da un lato non può sfuggire ad alcune necessità insite nella sua stessa natura (si pensi alla costruzione di un alveare, un nido, una tana; alla migrazione, al letargo, giusto per citare casi più familiari), poiché è costretto dal suo stesso essere ad agire in determinate maniere, senza intenzionalità e possibilità di scegliere altrimenti; dall'altro interviene deliberatamente e liberamente sulle modalità con cui esprimersi.

Se il ragno non può non tessere la ragnatela, perché necessitato dalla sua stessa natura, può tuttavia decidere come e dove realizzarla, manifestando in ciò forme di intelligenza riconducibili al discernimento. La macchina è invece mera reiterazione:

Gli animali adattano il loro modo di operare, che è determinato certo in generale, alle circostanze, arrangiandolo e modificandolo di

59 SCHOPENHAUER, A. Ivi, p. 53.

60 Ivi, p. 54.

Una possibile conferma postuma: punti di congiunzione tra Schopenhauer e Darwin 
conseguenza. La macchina, se la sua prima operazione è disturbata o rovinata, pure effettua tutte le operazioni successive, anche se in tal caso del tutto invano. L'uccello, invece, se noi rompiamo il nido che ha cominciato, lo ripara oppure ne fa un altro; lo adatta in generale alla natura del luogo. Se laceriamo il bozzolo che un bruco ha iniziato, esso lo rammenda due, tre volte, ne rappezza lo squarcio. Talvolta gli animali si confondono, sbagliano e quindi apportano correzioni, recuperano il tempo perso. [...] L'agire degli animali conformemente ad impulsi industriosi è, dunque, certamente accompagnato da conoscenza, ma non è guidato da essa, vale a dire non nel complesso, non conformemente allo scopo. [...] Ma essa entra in attività quando si tratta della dettagliata coordinazione dei mezzi, del loro adattamento alle circostanze di tempo e di luogo 61.

Dovrebbe evincersi che l'istinto non può risolversi esclusivamente né in un agire meccanico né meditato. È «impulso formativo rivolto al di fuori ${ }^{62} 》$, accompagnato dalla conoscenza. È, seppur con una certa prevalenza, qualcosa di composito, che unisce volontario e involontario, automatico e riflesso.

Pertanto, l'antitesi istinto intelligenza, che si pone come un aut-aut, è infondata, basandosi sulla non comprensione dell'incontro tra irriflesso e ponderato. L'agire istintivo, come è stato appena illustrato, comprende l'intervento della conoscenza, che si esplica in svariati modi. Parimenti, un agire con conoscenza non esclude il sussistere di istinti, all'interno del suo stesso corso e rispetto ad altre condizioni. Senz'altro si danno situazioni in cui prevale la riflessione o il suo contrario, ma questo non significa che non possano presentarsi entrambi, quasi all'unisono e in tempi diversi in uno stesso individuo, o che un atteggiamento non sia il risultato di ambedue i movimenti.

Darwin fornisce una spiegazione non dissimile da quella schopenhaueriana, riconoscendo come tendenza prevalente un obbedire inconsapevole alla necessità, a cui partecipa un agire consapevole guidato dalla riflessione.

Riepilogando con il naturalista inglese:

In tutti i casi in cui gli individui delle specie, in tutti i tempi conosciuti, in modi pressoché invariabili e senza conoscerne il fine, eseguono una azione senza l'aiuto dell'esperienza, istruzione o sufficiente capacità di ragionamento, laddove tali aiuti sarebbero necessari, queste circostanze sono state generalmente ritenute come le principali caratteristiche degli istinti63.

${ }^{61}$ Ivi, pp. 54-55.

62 SCHOPENHAUER, A. Metafisica della natura, p. 61.

63 DARWIN, C. Capacità mentali e istinti negli animali, pp. 6-7.

Una possibile conferma postuma: punti di congiunzione tra Schopenhauer e Darwin 
Si tratta di parole in cui non ci sembra improprio intravedere quelle del filosofo tedesco, quando afferma che l'agire istintivo appare guidato interamente dal concetto di fine, quindi dal conoscere, ma non lo è, poiché alcune azioni si svolgono senza aver avuto alcuna possibilità di osservare, apprendere e quindi imitare il comportamento altrui, altre senza aver conosciuto i luoghi in cui e le prede da cui nascondersi64. Inoltre, abbiamo constatato che Schopenhauer attribuisce la scelta dei mezzi da usare, l'adattare un comportamento alle circostanze, il decidere e valutare spazio e tempo idonei etc. all'intervento della conoscenza. Tutte azioni che rivelano uno dei tanti lati dell'intelligenza.

Anche Darwin sostiene qualcosa di analogo. Difatti, subito dopo il summenzionato passo, precisa con un'avversativa: «Tuttavia nessuna di queste caratteristiche può dirsi del tutto assoluta. Non c'è dubbio infatti che a volte la ragione entra in gioco nell'eseguire azioni istintive ${ }^{65} . »$ Più avanti: «Credo fermamente che gli istinti, per qualche lievissima gradazione, siano a volte soggetti all'influenza della ragione, dell'esperienza, dell'istruzione e dell'imitazione ${ }^{66} . »$

Forniamo ora qualche esempio, per far comprendere più da vicino il darsi congiunto di azione riflessa e irriflessa.

Si consideri l'incredibile istinto delle api nel costruire alveari. Esse non si adoperano per realizzare strutture diversificate con materiali variegati. Probabilmente non è data loro questa possibilità, costrette a obbedire alla propria natura confinata, che non consente di scegliere qualcosa di diverso da ciò che contiene e im-pone. L'ape non può decidere se costruire un alveare o un nido. Tuttavia la limitatezza del suo essere, determinata e determinante, non esclude al suo interno forme di indeterminatezza, cioè possibilità, seppur circoscritte, di trascegliere, prediligere, rifuggire. Per necessità le api architettano cellette esagonali perfette:

La semplice e casuale vista di un favo di api deve suscitare in tutti la più viva ammirazione. Quando poi veniamo a sapere che le api hanno risolto in pratica un problema geometrico di difficoltà massima, cioè quello di costruire le loro celle con il minimo consumo di cera, facendo esagoni alternativamente opposti tra loro, con basi formate di tre piani romboidali con angoli di $109^{\circ} 28^{\prime}$ e $70^{\circ} 32^{\prime}$, inclinati tra loro con angolo

64 SCHOPENHAUER, A. Metafisica della natura, pp. 54 ss.

65 DARWIN, C. Capacità mentali e istinti negli animali, p. 7.

66 Ivi, p. 21.

Una possibile conferma postuma: punti di congiunzione tra Schopenhauer e Darwin 
di $120^{\circ}$, angoli che i matematici hanno provato e verificato essere corretti, - allora, a vedere tutto ciò, la nostra ammirazione è messa a tacere dalla stupefazione ${ }^{67}$.

Contemporaneamente sono libere e in grado di valutare dove crearle, riconoscono imperfezioni costruttive e sanno in che modo risolvere eventuali difficoltà o apportare modifiche, legate a imprevisti e circostanze mutevoli. Ad esempio, demolire e costruire una celletta ripetutamente, affinché risulti confacente al suo uso, è un comportamento in cui un fare immeditato si mescola a stille di riflessione: non si dà alcuna scelta intenzionale nella costruzione, tuttavia ci si rende conto dell'errore o di un imprevisto e si agisce per rimediarvi, fino a quando non si riconosce una figura esagonale adatta.

Quindi, all'interno di un non poter essere e fare altrimenti, si inseriscono sfumature di libertà, basate su un certo grado di coscienza del circostante e di sé, a partire da cui si fondano forme di intelligenza che muovono dall'osservare al valutare, dallo scrutare all'imitare, dal contemplare al discernere e risolvere problemi. Non soltanto l'istinto non può definirsi unilateralmente, essendo qualcosa di composito, che include forme di intelligenza, ma anche quest'ultima non può risolversi in una descrizione univoca; è plurale e conosce innumerevoli gradazioni.

Come le api, anche le formiche operaie hanno abitazioni di cui si curano, meccanicamente e intelligentemente. Costruiscono e riparano nidi in modo automatico, procurano cibo, si occupano della difesa delle abitazioni senza mediazione-meditazione, ma la scelta dei mezzi per la sussistenza, la loro coordinazione, e la comunicazione reciproca, costituiscono qualcosa che si allontana dall'automatismo per avvicinarsi al mediato e meditato, che vaglia situazioni e soluzioni, le rende dicibili, conducendo a una tra le possibili risoluzioni e non a una scelta obbligata che in realtà scelta non può definirsi - poiché l'agire univoco è costrizione.

Proseguendo con l'istinto di costruire abitazioni, ammiriamo gli uccelli

\footnotetext{
${ }^{67} \mathrm{Ivi}$, pp. 91-92. È stato accennato che la selezione naturale opera anche rispetto agli istinti, conservando lentamente e instancabilmente quegli abiti vantaggiosi, che possono subire variazioni a seconda delle diverse condizioni, e che si configurano in modo preciso lentamente, trattandosi di lievi accumulazioni in un arco temporale vastissimo. Per quanto riguarda le api, Darwin parla della selezione naturale in relazione all'economia della cera: poiché il miele è fondamentale per il loro sostentamento, e da esso si ricava la cera, chi spreca minor cera, risparmia più miele, risultando così avvantaggiato rispetto a chi non riesce ad essere parsimonioso. - Risparmio che è strettamente legato alla forma esagonale e allo spessore delle cellette comunicanti reciprocamente, (e su di esso opera la selezione naturale, conservando e trasmettendo quei comportamenti favorevoli a tale risultato).
} 
affaccendarsi nel ricercare rametti, foglie e filamenti da intrecciare in modo perfetto, per accogliere le vite future. Anche se non sono stati istruiti su questo lavoro, sono in grado di realizzare focolari meravigliosi. Non hanno potuto osservare, per poi imitarla, la propria madre adoperarsi, poiché alla loro nascita era già tutto realizzato; non hanno potuto apprendere mediante l'esperienza come disporre i vari elementi, eppure ne sono altamente capaci. Di contro, attraverso essa valutano quali siano i luoghi più sicuri, quali i possibili pericoli da cui ripararsi e gli inconvenienti da evitare. Inoltre, se un nido viene modificato, da agenti atmosferici o possibili predatori, è probabile che gli uccelli giudichino opportuno allontanarsi dal luogo prescelto o decidano di fortificare il nido.

Sorprendente è anche la capacità del lumbricus terrestris di costruire e tappezzare gallerie. Si tratta di un anellide dalle piccole dimensioni, privo di vista e quasi sordo, ipersensibile al tatto e alla luce, tuttavia in grado di scavare cunicoli sicuri, nonché di comunicare, di scegliere il suo cibo e di riconoscere i suoi "coinquilini". Mediante il suo particolare processo digestivo, ricco di succhi e acidi nutrienti, è il principale creatore di humus. Inoltre, del tutto inconsapevolmente, custodisce antiche rovine. Soffermandoci sulla loro capacità di costruire abitazioni, possiamo notare che i lumbrici sono soliti creare gallerie, tappando le aperture con le foglie, riaprendole la sera. Stando alle osservazioni darwiniane, essi afferrano le foglie per lo più dalla punta, comportamento prevalente che non è esclusivo. Difatti, se si muovessero nello stesso modo rispetto ad ogni foglia, nota o ignota, potremmo dedurre che il loro fare è completamente privo di discernimento. Ma, poiché trascinano diversamente foglie diverse, possiamo supporre siano in grado di riconoscere forme distinte comportandosi di conseguenza, trasportando le foglie per le parti più convenienti. Ecco quindi un altro caso di mescolanza tra il meccanico e il riflesso 68 .

68 Circa quest'umile vivente, troppo spesso ignorato o maltrattato, si legga il mirabile ultimo scritto darwiniano: L'azione dei vermi nella formazione del terriccio vegetale con osservazioni sulle loro abitudini. Si tratta di un libro in cui viene ribadito quanto ciascun essere sia compiuto in sé e in relazione al suo microcosmo, poiché dotato di tutto ciò, o quasi, di cui ha bisogno. Viene sottolineato anche che si tratta di un animale portatore di vita, involontariamente, e che l'umano a maggior ragione dovrebbe salvaguardare. Non potendoci attardare come vorremmo su questo scritto, riportiamo due considerazioni sintetiche, di Pievani e di Scarpelli, che a nostro avviso compendiano ciò che quell'opera voleva suggerire, ben lungi dall'essere un «capriccio senile» o un'analisi irrisoria su viventi altrettanto insignificanti. Per Pievani si tratta di «un inno a quei 'meravigliosi dettagli' e ai 'fatti apparentemente insignificanti' grazie ai quali la natura esprime la sua creatività, un'illustrazione di come il reiterarsi di minuscole attività quotidiane (i movimenti e il metabolismo dei vermi) possa a lungo termine riuscire addirittura a modellare la campagna inglese. 'Ciò che è umile può spiegare ciò che è grande', scrisse in qui giorni. [...] L'umiltà del lombrico è un'ironica denuncia contro qualsiasi pretesa umana di sentirsi padroni della storia naturale 0 , ancor peggio, suo inevitabile compimento. Nelle creature più disprezzate e silenziose c'è il segreto dell'evoluzione, cioè 'gli effetti di una causa costantemente ricorrente'. Il messaggio della storia naturale 
Straordinario è anche l'istinto della migrazione, che guida verso nuove rotte come se l'animale fosse «provvisto di una bussola», riuscendo a calcolare distanze, a prevedere tempi cronici e uranici, momenti in cui attendere e ripartire. Tutt'oggi tali capacità non sono ancora perfettamente spiegate dall'umano. Probabilmente non saranno mai comprese interamente, profondamente, per la differenza percettivoorientativa che intercorre tra gli animali: ciò che è a tutti gli animali comune (nel caso specifico la percezione del tempo e dello spazio) assume comunque connotati peculiari, dando vita a uno degli innumerevoli punti di vista sulla realtà, costituendo uno dei tanti lati di cui la vita si compone69.

Composito e mirabile è anche l'istinto della simulazione della morte. È il risultato di più elementi appresi e trasmessi lentamente, successivamente. L'animale ha non solo un'immagine di sé, elevata astrattamente, ma è in grado di simulare la sua figura, morente. Paura e sospetto vengono acquisiti attraverso l'integrazione con l'uomo e con altri cacciatori, ma anche soccorrendo invano i propri compagni dolenti. Dalla vista della sofferenza altrui si originano empatia e riflessione, intelligenza ed emotività, individualità e comunità, tutti elementi racchiusi in tale istinto.

Dunque, alla luce di quanto detto, e di quanto si potrebbe ancora illustrare in una raccolta di più volumi, non bisognerebbe esitare nel riconoscere a tutti gli animali una coscienza-conoscenza, di sé e dell'altro; da cui derivano forme di intelligenza, dal

del lombrico era per lui più potente di qualsiasi sistema filosofico costruito a priori. Occorre uno sguardo laterale, non antropocentrico, e assai lungimirante per calcolare gli effetti a lungo termine del tempo, per avvertire la nostra posizione periferica e contingente nel grande albero della biodiversità passata e presente, per capire come la vita incessantemente rigenera se stessa nei cicli e ricicli della sua materia.» (PIEVANI, T. Introduzione a Darwin, pp. 138-139). Scarpelli invece domanda retoricamente: «L'umile anellide, nel suo ininterrotto procedere lungo budelli sotterranei, alla lunga genera, oltre all'agglomerarsi dell'humus e al dissodamento, la preservazione dei ruderi e la trasformazione del paesaggio. Tale accumularsi di operazioni impercettibili, che procura esiti tanto imprevedibili quanto grandiosi, che cosa richiama alla mente se non proprio l'immagine della selezione naturale, insostituibile occulta e inesausta, che nel suo plurimillenario viaggio ha tramutato il pianeta vivente? L'evoluzione è come il lombrico, cieca e paziente, e lega le specie con un'origine comune.» (SCARPELLI, G. Darwin e i cunicoli dell'evoluzione. In: DARWIN, C. L'azione dei vermi nella formazione del terriccio vegetale, con osservazioni sulle loro abitudini, p. 18).

${ }^{69}$ Prospettivismo che Nietzsche avrebbe rimarcato, muovendo dalle orme del suo educatore: «È evidente che ogni creatura diversa da noi percepisca altre qualità e quindi vive in un mondo diverso da quello in cui viviamo noi. Pretendere che queste nostre interpretazioni e valori umani siano valori universali e forse costitutivi è una pazzia ereditaria della superbia umana.» (NIETZSCHE, F. La volontà di potenza, frammento 565). Altrove, «[...] Già all'uomo costa molta fatica ammettere che l'insetto o l'uccello percepiscono un mondo del tutto diverso rispetto a quello dell'uomo, e che chiedersi quale sia la più giusta delle due percezioni è assolutamente privo di senso, poiché qui si dovrebbe misurare in base al paradigma della giusta percezione e cioè in base ad un paradigma che non esiste» (NIETZSCHE, F. Su verità $e$ menzogna in senso extramorale, p. 134). 
giudizio al ricordo, al sogno, all'immaginazione, alla scelta, alla risoluzione. Tale riconoscimento ne racchiude altri.

Muovendo dalla percezione del circostante, l'animale vive i moti emotivi più diversi. Per Schopenhauer, «l'animale ha tutti i sentimenti dell'uomo: gioia, tristezza, paura, collera, amore, odio, brama, invidia ecc. ${ }^{70}$ ». Può provare affetto o timore nei confronti dei suoi simili e dissimili, può gioire scodinzolando o sorridendo; per la paura o la vergogna può abbassarsi al suolo con la coda tra le gambe o coprirsi il volto con le mani; può dispiegare il piumaggio per il corteggiamento, modulare la voce e il canto. Per il filosofo è indiscutibile che tutti gli animali provino emozioni. Avendo riconosciuto in loro la volontà illuminata dal conoscere (e non la sua gradazione di forza naturale o forza vitale, del tutto prive di conoscenza) quindi un agire mosso da motivi, cause di cui si ha conoscenza, è evidente che questa conoscenza provochi affezioni oltreché pensieri. Precisamente, l'animale non umano possiede quella stessa essenza che caratterizza l'uomo e qualsiasi altro fenomeno naturale, mutando soltanto gli accidenti. Anche per questo motivo deve essere rispettato: «È giunta l'ora», ammonisce Schopenhauer,

di riconoscere, risparmiare e rispettare in quanto tale l'eterna essenza, che, come in noi, vive anche in tutti gli animali. Sappiatelo! Ricordatelo! È una cosa seria e non si transige. [...] Bisogna essere ciechi in tutti $\mathrm{i}$ sensi [...] per non riconoscere che l'animale, nelle cose essenziali e principali, è assolutamente la stessa cosa che siamo noi, e che la differenza sta soltanto nelle cose accidentali [...]. Il mondo non è un'opera raffazzonata, né gli animali sono prodotti di fabbrica per nostro uso e consumo ${ }^{71}$

Ora, se Schopenhauer si limita a constatare e ribadire una condizione, così lampante da non necessitare spiegazioni articolate, ma solo un'osservazione scevra di pregiudizi, Darwin dimostra in modo puntuale che tutti gli animali nutrono sentimenti,

\footnotetext{
${ }^{70}$ SCHOPENHAUER, A. N, p. 88.

${ }^{71}$ SCHOPENHAUER, A. P II, pp. 493-494. Inoltre, Schopenhauer si indignava molto contro coloro che maltrattano gli animali non lasciandoli vivere nelle condizioni adeguate, ritenendo che «questi maltrattamenti degli animali dovrebbero essere proibiti per legge» (Ivi, p. 496): «[...] L'uomo imprigiona in un metro cubo di spazio l'uccello che è organizzato per spaziare nella metà del mondo; qui rinchiuso, esso lentamente muore languendo e cantando, poiché: l'uccello nella gabbia canta non di piacere ma di rabbia, non solo, ma l'uomo mette alla catena il suo animale più fedele, il cane, che è così intelligente! Non mi capita mai di vederne uno senza provare grandissima compassione per il cane e profonda indignazione per il suo padrone, e con vera soddisfazione ripenso a un caso narrato alcuni anni fa dal "Times": un Lord, il quale teneva un bel cane alla catena, un giorno attraversando il giardino si azzardò ad accarezzarlo, ma il cane gli sbranò il braccio in tutta la lunghezza - ben fatto! Con ciò voleva dire: 'Tu non sei il mio padrone, ma il mio diavolo, che vuol trasformare in inferno la mia breve esistenza.' Possa ciò succedere a tutti quelli che mettono il cane alla catena.» Ivi, pp. 229-230. 
riprovando in tal modo la posizione del filosofo senza che questi riuscisse a saperlo, a conoscere i suoi scritti.

Come abbiamo detto all'inizio del saggio, poiché Schopenhauer ripone fiducia nelle scienze future, in merito a possibili punti di contatto tra esse e la sua metafisica, abbiamo considerato gli studi darwiniani sulle emozioni come conferma plausibile alle intuizioni schopenhaueriane; come pure quelli sugli istinti, e altri aspetti della vita, quali altrettante comprove. Potremmo aver errato intorno a tale "conferma a posteriori", non comprendendo pienamente il pensiero schopenhaueriano. In tal caso, ci auguriamo che il filosofo possa scusarci nell'intento: mostrare una visione che, nel tentativo di comprendere il reale, ha gettato sprazzi di luce «nello sconfinato buio originario».

Vediamo molto rapidamente alcuni nuclei tematici de L'espressione delle emozioni nell'uomo e negli animali, scorgendo riaffermazioni del pensiero del filosofo secondo cui: «[...] parliamo senza esitare della sua [dell'animale] brama, avversione, paura collera, odio, amore, gioia, afflizione, struggimento ${ }^{72}$.»

Nell'indagine intorno alle emozioni, le sue sfumature ed espressioni, Darwin analizza non soltanto quali espressioni si presentano in relazione alle emozioni, quando e come si manifestano, confrontando i condivisi moti animali, diversificantesi tra loro essenzialmente per il modo di esternarsi. Egli indaga anche intorno al perché i moti dell'animo si rivelano in una certa maniera, poiché desidera documentare e riaffermare l'unica grande rete intessuta tra i viventi. Quindi, oltre alle emozioni, molte estrinsecazioni sono simili perché condivisa è la discendenza.

Un mezzo espressivo comune, che svela un sentire affine, è il suono. Esso può comunicare astrazioni e necessità, ira e paura, timore e amore. Plurali sono le sue tonalità e articolazioni: può manifestarsi come canto e ululato, potpottio e squittio, gorgoglio e bubbolio, grugnito e latrato, ruggito e soffio; può diventare linguaggio umano. Il gloglottare del tacchino e il barrire dell'elefante, il paupolare del pavone e il miagolare del gatto, il pregare e il dialogare sono tutte forme vocali diversissime che dicono anche moti affini. L'origine stessa del suono sembrerebbe risalire alla necessità di comunicare stati d'animo e bisogni, pericoli imminenti, attività pragmatiche, e non concetti (elemento successivo che indiscutibilmente distingue, per la sua complessità, la

\footnotetext{
72 Altrimenti detto, «[...] un desiderare, bramare, volere, o un aborrire, rifuggire, non volere, sono propri di ogni coscienza: l'uomo li ha in comune col polpo. Ciò è dunque l'essenziale e la base di ogni coscienza» (SCHOPENHAUER, A. WWV II, pp. 1387-1389).

Una possibile conferma postuma: punti di congiunzione tra Schopenhauer e Darwin
} 
sonorità umana da quella animale).

Così argomenta Darwin:

Può darsi che l'emissione di suoni vocali abbia preso origine dalle contrazioni involontarie e prive di finalità dei muscoli del torace o della glottide eccitati, come si è visto in precedenza, dal dolore o dalla paura. Ma attualmente la voce è molto usata da numerosi animali per vari scopi; e si ha l'impressione che l'abitudine abbia avuto una funzione importante nella sua utilizzazione in altre circostanze. [...] Questo significa che la voce, essendo stata usata abitualmente come utile aiuto in diverse condizioni che inducevano piacere, dolore, rabbia ecc., verrà ora impiegata comunemente ogni qualvolta vengano suscitate quelle stesse sensazioni o emozioni, quand'anche esse si presentino in situazioni completamente differenti o con intensità inferiori ${ }^{73}$.

Quindi, lo sviluppo della voce deriverebbe dal suo uso ripetuto, volontario, appena scorta la sua utilità, a partire da quelle prime circostanze che l'hanno provocata inavvedutamente. Un aspetto connesso all'elemento sonoro è la vastità del patire, che implica quasi tanti suoni quanti sono i sentimenti. Le fusa alludono a una certa contentezza, o a un volersi rassicurare, l'ululato è un richiamo o un'espressione di struggimento, i cinguettii e i pigolii sono forme di racconto, il ringhio ha significato offensivo e difensivo, l'emettere suoni con gli zoccoli, scalciando, rappresenta un'avvisaglia, ma un affine scalpitare è indice di gioia. Le api ronzano in modo diverso a seconda del messaggio da riportare, molti uccelli battono il becco o producono suoni attraverso il piumaggio. Segnali acustici e ultrasuoni accomunano gli animali marini, suoni impercettibili o quasi all'orecchio umano appartengono al mondo degli insetti. Si ha così una sorprendente sinfonia comunicativa ${ }^{74}$.

Oltre alla sonorità, un altro modo, comune, per esprimere il sentimento dell'affetto è il contatto. Al contrario, la disaffezione è espressa dal distacco.

Esseri umani e animali tendono ad avvicinarsi ad altri viventi se provano un

73 DARWIN, C. L'espressione delle emozioni nell'uomo e negli animali, p. 124.

${ }^{74}$ Leggiamo una descrizione darwiniana: «Gli animali che vivono in società, quando sono separati, spesso si chiamano l'un l'altro, ed è evidente che provano una grande contentezza quando si ritrovano, lo possiamo vedere in un cavallo, quando si riunisce al compagno che chiamava nitrendo. La madre che ha perso i suoi cuccioli li chiama continuamente, come succede per la mucca che ha perso il vitellino; e i piccoli di molti animali usano chiamare la madre. Quando un branco di pecore viene disperso, le femmine belano in continuazione per richiamare gli agnelli, ed è ben visibile il piacere che provano sia le une sia gli altri quando si ritrovano insieme. Rischia molto l'uomo che si avvicina ai cuccioli dei quadrupedi più grandi e feroci, se questi sentono il grido di angoscia dei loro piccoli. La rabbia provoca la contrazione violenta di tutti i muscoli, compresi quelli della fonazione; e alcuni animali, quando sono arrabbiati, cercano di terrorizzare i loro nemici producendo suoni fortissimi e acuti, come fa il leone con i suoi ruggiti, il cane abbaiando ecc.»Ivi, p. 125.

Una possibile conferma postuma: punti di congiunzione tra Schopenhauer e Darwin 
sentimento di simpatia e amorevolezza. Certo, possono approssimarsi anche per monitorare una situazione o per attaccare, ma in questa circostanza ci interessa mostrare come una carezza o un morsetto siano riconducibili ad uno stesso moto. Un bacio è vicinanza spaziale che esprime quella individuale, implicando un certo sentire: affinità, accoglienza, condivisione, amore, rassicurazione, empatia e altro ancora. Significa essere vicini all'altro metaforicamente. Anche il leccarsi animale indica qualcosa di analogo. Una madre inumidisce i suoi cuccioli non soltanto per pulirli, per istinto ancestrale, ma soprattutto per comunicare le sue premure. Animali umani e non umani si abbracciano. Le parti corporee con cui avviene ciò non sono propriamente le stesse, ma l'essenza di quell'agire è immutata. È di secondaria importanza che a incrociarsi siano quadrupedi o bipedi, che a sfiorarsi siano mani o zampe, nella misura in cui si tratta di differenze solo strutturali (d'altronde riflettenti un comune modello) attraverso cui si esprime un comune sentire. Il vivente uomo accarezza i suoi cari sul volto o dà piccoli colpetti con la mano, affini alle lievi testate di altri animali, che accarezzano strofinandosi con insistenza intorno ai corpi amici ${ }^{75}$. Schopenhauer, che amava soprattutto i cani, e ne aveva uno di nome Atma, era solito accarezzarlo continuamente, lodando anche la sua «trasparenza ${ }^{76} »$.

Il sentire opposto all'affetto si esterna con atteggiamenti contrari a quelli appena esaminati. Tali condotte, di avvicinamento e allontanamento, sono riscontrabili finanche nelle piante, che esprimono in quel modo simpatia e antipatia, attrazione e repulsione, gradimento e fastidio, ad esempio in relazione ad appigli o altre piante ${ }^{77}$. Per usare termini molto generici, per indicare un atteggiamento che indica per lo più quanto detto, diciamo che l'accostarsi rimanda al piacevole e il ritrarsi allo spiacevole, il contatto all'affetto e il distacco alla disaffezione. Sebbene, sia chiaro, anche la distanza dello sguardo possa dire l'amore.

\footnotetext{
75 Pertinenti a riguardo le parole darwiniane: «Normalmente si prova un forte desiderio di toccare la persona amata, e questo gesto esprime l'amore più chiaramente di qualsiasi altro. Così desideriamo ardentemente stringere fra le braccia la persona che amiamo teneramente. Forse questo desiderio ci viene da un'abitudine ereditaria, in associazione con l'abitudine a nutrire e a prendersi cura dei propri figli e con quella delle reciproche carezze degli amanti. Negli animali inferiori all'uomo constatiamo lo stesso fatto, cioè il piacere che deriva dal contatto, in associazione con l'affetto. È evidente che i cani provano piacere a strofinarsi contro i loro padroni e le loro padrone e a riceverne carezze e colpetti affettuosi [...].» Ivi, p. 244. Strofinarsi che, nel caso dei gatti, è accompagnato dalla coda eretta e dalle fusa. Inoltre mordicchiano e leccano. I cani invece scodinzolano (la coda ritta indicherebbe un'emozione contraria), saltano e cercano contatti con il loro padrone.

76 A riguardo, si legga un racconto riportato da Julius Frauenstädt. In: SCHOPENHAUER, A. Colloqui, p. 100. ${ }_{77}$ Cfr. DARWIN, C. Piante a viticci. In: DARWIN, C. I movimenti e le abitudini delle piante rampicanti.

Una possibile conferma postuma: punti di congiunzione tra Schopenhauer e Darwin
} 
Unitamente alle affini emozioni ed estrinsecazioni, il patire può esternarsi anche in modi del tutto peculiari. Ad esempio, le lacrime sono propriamente umane, come l'arrossire, ma questo non implica che altri animali non provino imbarazzo, vergogna, forme di dolore o commozione connesse a queste manifestazioni. Viceversa, l'uomo non sa comunicare attraverso i movimenti delle orecchie, talvolta impercettibili a un occhio inesperto nell'interpretarli. - Le orecchie spinte indietro con forza possono indicare spavento o una disposizione aggressiva; le orecchie tese implicano una certa attenzione; leggermente piegate all'indietro oppure rilassate denotano una situazione di piacevolezza. - L'uomo non può comunicare attraverso il pelo, piume e aculei, che non ha, abbondantemente usati dagli altri animali, per il corteggiamento, per incutere timore, per difesa, per delimitare il territorio.

Il mondo emotivo rivela sentimenti comuni e manifestazioni condivise, emozioni particolari ed espressioni peculiari, afferma e disvela l'identico nel diverso e il diverso nell'identico. Le forme corporee, gli embrioni, gli organi rudimentali sembrano suggerirci qualcosa di analogo. Simboleggiano l'unità della discendenza, la discendenza comune con modificazione. Evocano l'unità e identità del principio vitale: il Wille che anela a perdurare. Appulsione instancabile. E che desidera ovunque la stessa cosa, la vita.

\section{Riferimenti bibliografici}

ASHER, D. Schopenhauer and Darwinism. In: The Journal of Anthropology, Vol. 1, N. 3 (Jan., 1871), pp. 312-332. London: Royal Anthropological Institute of Great Britain and Ireland, 1871.

ATTANASIO, A. Introduzione - la «nuova» teoria dell'istinto di Darwin: natura, cultura, storia. In: DARWIN, C. Capacità mentali e istinti negli animali. A cura di Alessandra Attanasio. Torino: UTET università, 2011.

BACCELLI, V. Evoluzione e vita in Schopenhauer e Bergson. Gorgonzola: Aletti editore, 2016.

BELLINGRERI, A. La metafisica tragica di Schopenhauer. Milano: FrancoAngeli, 1992.

COLLI, G. prefazione a Arthur Schopenhauer, La quadruplice radice del principio di ragion sufficiente. In: COLLI, G. Per una enciclopedia di autori classici. Milano: Adelphi, 1995.

DARWIN, C. L'origine delle specie. Abbozzo del 1842. Lettere 1844-1858. Comunicazione del 1858. A cura di Telmo Pievani, tr. it. di Isabella C. Blum. Torino: Einaudi, 2009.

. L'origine delle specie. Tr. it. di Giuliano Pancaldi. Milano: BUR, 2010.

L'origine dell'uomo e la scelta in rapporto col sesso. Tr. it. di Michele Lessona. Milano: A. Barion, 1926.

L'espressione delle emozioni nell'uomo e negli animali. Terza edizione con introduzione, postfazione e commenti di Paul Ekman e con un saggio sulla storia delle illustrazioni di Philip Prodger, traduzioni di Fiamma Bianchi Bandinelli e Isabella C. Blum. Torino: Bollati Boringhieri, 2012.

Piante a viticci. In: DARWIN, C. I movimenti e le abitudini delle piante rampicanti. Tr. it. di Giovanni Canestrini e P. A. Saccardo, formato Kindle. Torino: Unione Tipografica-editrice, 1878.

Lettere sulla religione. A cura di Telmo Pievani, traduzione e note di Isabella C. Blum. Torino: 
Einaudi, 2013.

GOULD, S. J. La struttura della teoria dell'evoluzione. A cura di Telmo Pievani. Torino: Codice Edizioni, 2003.

LOVEJOY, A. O. Schopenhauer as an evolutionist. In: GLASS OWSEI TEMKIN H. B., STRAUSS, W. L. Forerunners of Darwin 1745-1859. Baltimora: J. Hopkins Press, 1959.

MANN, T. Schopenhauer. In: MANN, T. Nobiltà dello spirito e altri saggi. A cura di Andrea Landolfi, con un saggio di Claudio Magris, traduzioni di Bruno Arzeni, Italo Alighiero Chiusano, Enrico Gianni, Andrea Landolfi, Lavinia Mazzucchetti, Ervino Pocar, Adele Rossi. Milano: Mondadori, 1997.

MAYR, E. Storia del pensiero biologico. Diversità, evoluzione, eredità. A cura di P. Corsi. Torino: Bollati Boringhieri, 2011.

NIETZSCHE, F. Su verità e menzogna in senso extramorale. Prefazione di Gianni Vattimo, traduzione di Sossio Giametta. Milano: BUR 2009.

La volontà di potenza. A cura di Maurizio Ferraris e Pietro Kobau, tr. it. di Angelo Treves. Milano: Bompiani, 2008.

NOIRÉ, L. Der monistische Gedanke. Eine Concordanz der Philosophie Schopenhauer's, Darwin's, R. Mayer's und L. Geiger's. Leipzig: Veit, 1875.

PIEVANI, T. Creazione senza Dio. Torino: Einaudi, 2006.

Introduzione a Darwin. Roma-Bari: Laterza, 2012.

SCARPELLI, G. Darwin e i cunicoli dell'evoluzione. In DARWIN, C. L'azione dei vermi nella formazione del terriccio vegetale, con osservazioni sulle loro abitudini, a cura di Giacomo Scarpelli. Milano-Udine: Mimesis Edizioni 2012.

SCHOPENHAUER, A. Gesammelte Briefe. Herausgegeben von Arthur Hübscher. Bonn: Verlag Bouvier Herbert Grundmann, 1978.

Il mondo come volontà e rappresentazione, volume I e II. Introduzione e traduzione di Sossio Giametta, bibliografia e indici di Vincenzo Cicero. Milano: Bompiani, 2010. Sulla volontà nella natura. A cura di Sossio Giametta. Milano: BUR, 2010.

Parerga e Paralipomena. Scritti filosofici minori. Tomo primo a cura di Giorgio Colli, tomo secondo a cura di Mario Carpitella, tr. it. di Mazzino Montinari e Eva Kühn Amendola. Milano: Adelphi, 2007.

_.. Colloqui. Prefazione, traduzione e commento di Anacleto Verrecchia. Milano: BUR, 2000.

Metafisica della natura. A cura di Ignazio Volpicelli. Roma-Bari: Laterza, 2007.

Metafisica dei costumi. A cura di Maria Giovanna Franch. Milano: SE, 2008.

O si pensa o si crede. Scritti sulla religione. Introduzione di Anacleto Verrecchia, traduzione di

Bettino Betti e Anacleto Verrecchia. Prima edizione digitale. Milano: BUR, 2013.

SEGALA, M. Schopenhauer, la filosofia, le scienze. Pisa: Edizioni della Normale, 2009.

TEUTSCH, G. Reading Schopenhauer in the light of Present-Day Science. In: SchopenhauerJahrbuch, 93. Frankfurt am Main: Königshausen \& Neumann, 2012.

VOLPICELLI, I. A. Schopenhauer. La natura vivente e le sue forme. Settimo milanese: Marzorati editore, 1988.

VECCHIOTTI, I. Introduzione a Schopenhauer. Roma-Bari: Laterza, 2011.

WENG, G. Schopenhauer-Darwin: Pessimismus oder Optimismus?. Berlin: E. Hoffmann \& Company, 1911.

Recebido: $22 / 11 / 16$

Received: $11 / 22 / 16$

Aprovado: $01 / 06 / 17$

Approved: 06/01/17 